Алгебра и анализ

Том 20 (2008), № 4
St. Petersburg Math. J.

Vol. 20 (2009), No. 4, Pages 593-624

S 1061-0022(09)01063-2

Article electronically published on June 1, 2009

\title{
GENERALIZED FESENKO RECIPROCITY MAP
}

\author{
K. I. IKEDA AND E. SERBEST
}

\begin{abstract}
The paper is a natural continuation and generalization of the works of Fesenko and of the authors. Fesenko's theory is carried over to infinite APF Galois extensions $L$ over a local field $K$ with a finite residue-class field $\kappa_{K}$ of $q=p^{f}$ elements, satisfying $\boldsymbol{\mu}_{p}\left(K^{\text {sep }}\right) \subset K$ and $K \subset L \subset K_{\varphi^{d}}$, where the residue-class degree $\left[\kappa_{L}: \kappa_{K}\right]$ is equal to $d$. More precisely, for such extensions $L / K$ and a fixed Lubin-Tate splitting $\varphi$ over $K$, a 1-cocycle

$$
\boldsymbol{\Phi}_{L / K}^{(\varphi)}: \operatorname{Gal}(L / K) \rightarrow K^{\times} / N_{L_{0} / K} L_{0}^{\times} \times U_{\widetilde{\mathbb{X}}(L / K)}^{\diamond} / Y_{L / L_{0}}
$$

where $L_{0}=L \cap K^{n r}$, is constructed, and its functorial and ramification-theoretic properties are studied. The case of $d=1$ recovers the theory of Fesenko.
\end{abstract}

Let $K$ be a local field (that is, a complete discrete valuation field) with a finite residueclass field $\kappa_{K}$ of $q=p^{f}$ elements. Assume that $\boldsymbol{\mu}_{p}\left(K^{\mathrm{sep}}\right) \subset K$. We fix a Lubin-Tate splitting $\varphi$ over $K$ (see [10]). In [1, 2, 3], Fesenko introduced a very general non-Abelian local reciprocity map

$$
\Phi_{L / K}^{(\varphi)}: \operatorname{Gal}(L / K) \rightarrow U_{\widetilde{\mathbb{X}}(L / K)}^{\diamond} / Y_{L / K}
$$

defined for any totally ramified infinite APF Galois extension $L / K$ satisfying $K \subset L \subset$ $K_{\varphi}$, which generalized the earlier non-Abelian local class field theories of Koch and de Shalit [10] and Gurevich [7]. In 8], we studied the basic functorial and ramificationtheoretic properties of the reciprocity map of Fesenko.

In this paper, which is a natural continuation and generalization of [1, 2, 3, and 8], we extend the theory of Fesenko to infinite APF Galois extensions $L / K$ satisfying $K \subset L \subset K_{\varphi^{d}}$, where $d$ is the residue-class degree $\left[\kappa_{L}: \kappa_{K}\right]$. More precisely, for such extensions $L / K$, we construct a 1 -cocycle,

$$
\boldsymbol{\Phi}_{L / K}^{(\varphi)}: \operatorname{Gal}(L / K) \rightarrow K^{\times} / N_{L_{0} / K} L_{0}^{\times} \times U_{\widetilde{\mathbb{X}}(L / K)}^{\diamond} / Y_{L / L_{0}},
$$

where $L_{0}=L \cap K^{n r}$, and study its functorial and ramification-theoretic properties. Note that the case where $d=1$ recovers the theory of Fesenko.

The organization of this paper is as follows. In the first section, we briefly review the necessary background material from the Fontaine-Wintenberger theory of fields of norms. In the second section, we introduce the generalized Fesenko reciprocity map $\boldsymbol{\Phi}_{L / K}^{(\varphi)}$ of an extension $L / K$ that is an infinite APF Galois extension satisfying $K \subset L \subset K_{\varphi^{d}}$, where the residue-class degree $\left[\kappa_{L}: \kappa_{K}\right]$ is equal to $d$, and study its functorial and ramification-theoretic properties.

The material and results of this paper play a fundamental role in our construction of non-Abelian local class field theory [9], which generalizes also the Laubie theory [11].

2000 Mathematics Subject Classification. Primary 11S37.

Key words and phrases. Local fields, higher-ramification theory, APF extensions, Fontaine-Wintenberger field of norms, Fesenko reciprocity map, generalized Fesenko reciprocity map, non-Abelian local class field theory. 
Notation. Throughout this paper, $K$ will denote a local field (a complete discrete valuation field) with finite residue field $O_{K} / \mathfrak{p}_{K}=: \kappa_{K}$ of $q_{K}=q=p^{f}$ elements, where $p$ is a prime number; here $O_{K}$ denotes the ring of integers in $K$ with a unique maximal ideal $\mathfrak{p}_{K}$. Let $\boldsymbol{\nu}_{K}$ denote the corresponding normalized valuation on $K$ (normalized by $\boldsymbol{\nu}_{K}\left(K^{\times}\right)=\mathbb{Z}$ ), and let $\widetilde{\boldsymbol{\nu}}$ be the (unique) extension of $\boldsymbol{\nu}_{K}$ to a fixed separable closure $K^{\text {sep }}$ of $K$. For any subextension $L / K$ of $K^{\text {sep }} / K$, the normalized form of the valuation $\left.\widetilde{\boldsymbol{\nu}}\right|_{L}$ on $L$ will be denoted by $\boldsymbol{\nu}_{L}$. As usual, we let $K^{n r}$ denote the maximal unramified extension in $K^{\text {sep }}$, and $\widetilde{K}$ denotes the completion of $K^{n r}$ with respect to $\boldsymbol{\nu}_{K^{n r}}$. We fix a Lubin-Tate splitting $\varphi_{K}=\varphi$ over $K$. The fixed field of the Lubin-Tate splitting $\varphi$ is denoted by $K_{\varphi}$. Finally, let $\left(\pi_{E}\right)_{K \subset E \subset K_{\varphi}}$ be the canonical sequence of norm-compatible prime elements in finite subextensions $E / K$ in $K_{\varphi} / K$. This determines a unique LubinTate labeling over $K$ (see Subsection 0.2 in [10]).

\section{§1. Preliminaries on the Fontaine-Wintenberger field of norms}

For a brief review of APF extensions and the Fontaine-Wintenberger field of norms, we refer the reader to [8], and for detailed proofs to [5, 6, 12,

Let $L / K$ be an infinite, Galois, arithmetically profinite (in short APF) extension such that the residue-class degree $\left[\kappa_{L}: \kappa_{K}\right]$ is equal to $d$ and $K \subset L \subset K_{\varphi^{d}}$; in the terminology of Koch and de Shalit in [10] and Laubie in [11], $L$ is compatible with $(T, \varphi)$, where $T$ denotes the intersection field $L \cap K_{\varphi}$. Note that, in general, $T / K$ is not a normal extension! We denote $L_{0}^{(K)}=L \cap K^{n r}=K_{d}^{n r}$. If no confusion is possible, we denote $L_{0}^{(K)}$ simply by $L_{0}$. So, we have the following diagram:

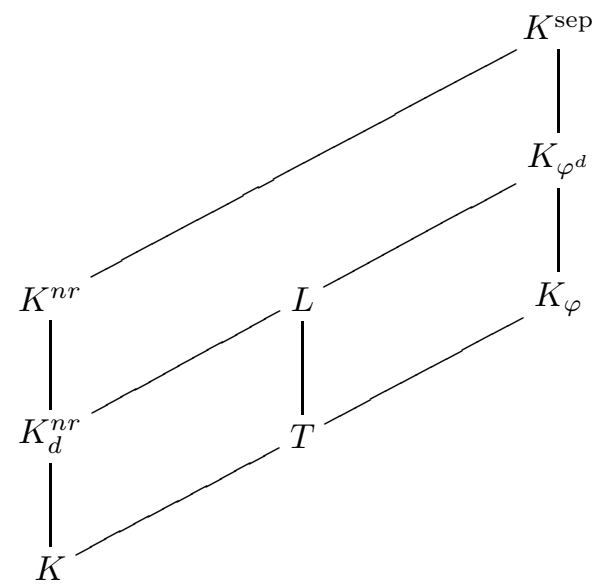

Remark 1.1. Note that $\varphi^{\prime}=\varphi^{d}$ is a Lubin-Tate splitting over $L_{0}=K_{d}^{n r}$. Therefore, by Proposition 1.2.3 in 12 or by Lemma 3.3 in $8, L / L_{0}$ is an infinite totally ramified APF Galois extension satisfying $L_{0} \subseteq L \subseteq\left(L_{0}\right)_{\varphi^{\prime}}$. Thus, the Fesenko theory developed in [1, 2, 3] and [8] works for the extension $L / L_{0}$.

Since $L / T$ is an unramified extension, the following statement is true.

Lemma 1.2. The field of norms $\mathbb{X}\left(L / L_{0}\right)$ is an unramified extension of the field of norms $\mathbb{X}(T / K)$.

Proof. In fact, there exists a natural isomorphism $\mathbb{X}\left(L / L_{0}\right) \stackrel{\sim}{\longrightarrow} \mathbb{X}(L / K)$ that identifies $\mathbb{X}\left(L / L_{0}\right)$ and $\mathbb{X}(L / K)$ (see Subsection 5.6 of Chapter III in [4]). Now, $\mathbb{X}(L / K)$ is a Galois extension of $\mathbb{X}(T / K)$ with the corresponding Galois group isomorphic to $\operatorname{Gal}(L / T)$ (see 
[8] and [12]). Since $\kappa_{\mathbb{X}(L / K)} \simeq \kappa_{L}$ and $\kappa_{\mathbb{X}(T / K)} \simeq \kappa_{T}$, it follows that

$$
\left[\kappa_{\mathbb{X}(L / K)}: \kappa_{\mathbb{X}(T / K)}\right]=[\mathbb{X}(L / K): \mathbb{X}(T / K)],
$$

because $L / T$ is an unramified extension, which proves that $\mathbb{X}(L / K)$ is an unramified extension of $\mathbb{X}(T / K)$.

Now, since the Lubin-Tate splitting $\varphi$ over $K$ is fixed, it follows that the element $\Pi_{\varphi ; T / K}=\left(\pi_{E}\right)_{K \subset E \subset T} \in \mathbb{X}(T / K)$ is a canonical prime element of the local field $\mathbb{X}(T / K)$. Thus, by Lemma 1.2. $\Pi_{\varphi ; T / K}$ is a prime element of $\mathbb{X}\left(L / L_{0}\right)$ as well. Moreover, the following is true.

\section{Lemma 1.3.}

$$
\Pi_{\varphi ; T / K}=\Pi_{\varphi^{\prime} ; L / L_{0}} .
$$

Proof. Indeed, for the Lubin-Tate splitting $\varphi^{\prime}=\varphi^{d}$ over $L_{0}=K_{d}^{n r}$, there exists a unique element $\left(\pi_{L_{0} E}\right)_{L_{0} \subset L_{0} E \subset L_{0} T=L} \in \mathbb{X}\left(L / L_{0}\right)$. Since $L_{0} E / E$ is an unramified extension, it follows that $\pi_{L_{0} E}=\pi_{E}$ for each $K \subset E \subset T$. Thus, (1.1) is fulfilled.

The completion $\widetilde{\mathbb{X}}(L / K)$ of the maximal unramified extension $\mathbb{X}(L / K)^{n r}$ of the field of norms $\mathbb{X}(L / K)$ is identified with the field of norms $\mathbb{X}(\widetilde{L} / \widetilde{K})=\mathbb{X}\left(\widetilde{L} / \widetilde{L_{0}}\right)$.

\section{§2. Generalized Fesenko Reciprocity map}

The main references for this section are [1, 2, 3] and [8.

Fix a Lubin-Tate splitting $\varphi_{K}=\varphi$ over $K$. Our aim in this section is to generalize the reciprocity map $\Phi_{M / K}^{(\varphi)}$ of Fesenko, see [1, 2, 3] and [8], defined for infinite totally ramified APF Galois extensions $M / K$ satisfying $K \subset M \subset K_{\varphi}$, to infinite APF Galois extensions $L / K$ with residue-class degree $\left[\kappa_{L}: \kappa_{K}\right]=d$ and satisfying $K \subset L \subset K_{\varphi^{d}}$. In what follows we shall keep the notation introduced in 8 and in the preceding section.

We recall that, for the extension $M / K$ as above, the diamond subgroup $U_{\widetilde{\mathbb{X}}(M / K)}^{\diamond}$ of the group $U_{\widetilde{\mathbb{X}}(M / K)}$ of units in the ring of integers of $\widetilde{\mathbb{X}}(M / K)$ is defined by

$$
U_{\widetilde{\mathbb{X}}(M / K)}^{\diamond}=\operatorname{Pr}_{\widetilde{K}}^{-1}\left(U_{K}\right),
$$

where $\operatorname{Pr}_{\widetilde{K}}: U_{\widetilde{\mathbb{X}}(M / K)} \rightarrow U_{\widetilde{K}}$ denotes the projection map on the $\widetilde{K}$-coordinate of $U_{\widetilde{\mathbb{X}}(M / K)}$. More generally, for a given infinite APF Galois extension $L / K$ with residue-class degree $\left[\kappa_{L}: \kappa_{K}\right]=d$ and satisfying $K \subset L \subset K_{\varphi^{d}}$, the diamond subgroup $U_{\widetilde{\mathbb{X}}(L / K)}^{\diamond}$ of the group $U_{\widetilde{\mathbb{X}}(L / K)}$ of units in the ring of integers of $\widetilde{\mathbb{X}}(L / K)=\widetilde{\mathbb{X}}\left(L / L_{0}\right)$ can be defined naturally as follows.

Definition 2.1. $U_{\widetilde{\mathbb{X}}(L / K)}^{\diamond}$ is the subgroup of the group $U_{\widetilde{\mathbb{X}}(L / K)}$ of units in the ring of integers of the local field $\widetilde{\mathbb{X}}(L / K)$ whose $\widetilde{K}=\widetilde{L}_{0}$-coordinate belongs to $U_{L_{0}}$. That is,

$$
U_{\widetilde{\mathbb{X}}(L / K)}^{\diamond}=U_{\widetilde{\mathbb{X}}\left(L / L_{0}\right)}^{\diamond}
$$

In Fesenko's theory, which was described in [1, 2, 3] and in detail in $\S 5$ of [8], an arrow $\phi_{M / K}^{(\varphi)}$ was defined for the extensions $M / K$, where $M / K$ is a totally ramified APF Galois extension satisfying $K \subset M \subset K_{\varphi}$. Now, as a first step, we generalize this arrow to infinite APF Galois extensions $L$ of $K$ having residue-class degree $d$ and satisfying $K_{d}^{n r} \subset L \subset K_{\varphi^{d}}$, and construct a generalized arrow $\phi_{L / K}^{(\varphi)}$ for such extensions $L / K$ as follows. There exists an isomorphism

$$
\operatorname{Gal}(L / K) \stackrel{\sim}{\longrightarrow} \operatorname{Gal}\left(L_{0} / K\right) \times \operatorname{Gal}\left(L / L_{0}\right)
$$


defined by

$$
\sigma \mapsto\left(\left.\sigma\right|_{L_{0}}, \varphi^{-m} \sigma\right)
$$

for every $\sigma \in \operatorname{Gal}(L / K)$, where $\left.\sigma\right|_{L_{0}}=\varphi^{m}$ for some $0 \leq m \in \mathbb{Z}$.

Remark 2.2. (i) Let $M / K$ be a Galois subextension of $L / K$. Let $M_{0}=M \cap K^{n r}$. Then, the square

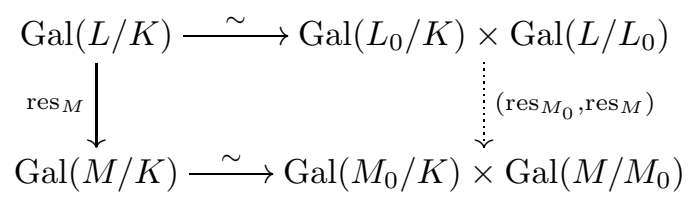

is commutative. Now, for $\sigma \in \operatorname{Gal}(L / K)$, we can find $0 \leq m, m^{\prime} \in \mathbb{Z}$ such that $\left.\sigma\right|_{L_{0}}=\varphi^{m}$ and $\left.\left(\left.\sigma\right|_{M}\right)\right|_{M_{0}}=\varphi^{m^{\prime}}$. Thus, $\left.\varphi^{m}\right|_{M_{0}}=\left.\varphi^{m^{\prime}}\right|_{M_{0}}$ and the identity $\left.\left(\varphi^{-m} \sigma\right)\right|_{M}=\varphi^{-m^{\prime}}\left(\left.\sigma\right|_{M}\right)$ is satisfied.

(ii) Let $F / K$ be a finite Galois subextension of $L / K$. Suppose $L_{0}^{(K)}=L \cap K^{n r}$ and $L_{0}^{(F)}=L \cap F^{n r}$. Then, the square

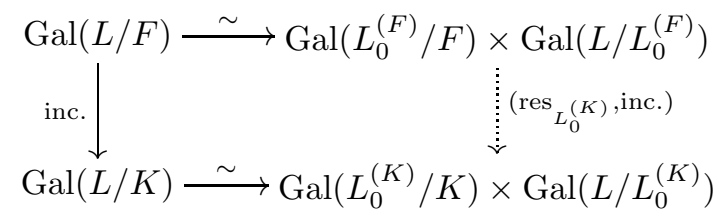

is commutative. Now, for any $\sigma \in \operatorname{Gal}(L / F)$, we can find $0 \leq m, m^{\prime} \in \mathbb{Z}$ such that $\left.\sigma\right|_{L_{0}^{(F)}}=\varphi_{F}^{m}$ and $\left.\sigma\right|_{L_{0}^{(K)}}=\varphi_{K}^{m^{\prime}}$. Thus, $\left.\varphi_{F}^{m}\right|_{L_{0}^{(K)}}=\varphi_{K}^{m^{\prime}}$ and the identity $\varphi_{F}^{-m} \sigma=\varphi_{K}^{-m^{\prime}} \sigma$ is satisfied.

By Proposition 1.2.3 in 12] or by Lemma 3.3 in [8, $L / L_{0}$ is a totally ramified APF Galois extension with $L_{0} \subset L \subset\left(L_{0}\right)_{\varphi^{\prime}}$, where $\varphi^{\prime}=\varphi^{d}$ is a Lubin-Tate splitting over $L_{0}$ by Remark 1.1. Thus, the map

$$
\phi_{L / K}^{(\varphi)}: \operatorname{Gal}(L / K) \rightarrow K^{\times} / N_{L_{0} / K} L_{0}^{\times} \times U_{\widetilde{\mathbb{X}}(L / K)}^{\diamond} / U_{\mathbb{X}(L / K)}
$$

can be defined by

$$
\boldsymbol{\phi}_{L / K}^{(\varphi)}(\sigma):=\left(\pi_{K}^{m} \cdot N_{L_{0} / K} L_{0}^{\times},\left(u_{\widetilde{E}}\right) \cdot U_{\mathbb{X}(L / K)}\right),
$$

where $\sigma \in \operatorname{Gal}(L / K)$ is such that $\left.\sigma\right|_{L_{0}}=\varphi^{m}$ for some $0 \leq m \in \mathbb{Z}$, and $U=\left(u_{\widetilde{E}}\right) \in$ $U_{\widetilde{\mathbb{X}}\left(L / L_{0}\right)}$ satisfies

$$
U^{1-\varphi^{d}}=\Pi_{\varphi^{\prime} ; L / L_{0}}^{\left(\varphi^{-m} \sigma\right)-1}
$$

where $\Pi_{\varphi^{\prime} ; L / L_{0}}$ is the canonical prime element of the local field $\mathbb{X}\left(L / L_{0}\right)$, which is the canonical prime element $\Pi_{\varphi ; T / K}$ of the local field $\mathbb{X}(T / K)$ by Lemmas 1.2 and 1.3 . Thus, (2.5) can be reformulated as

$$
U^{1-\varphi^{d}}=\Pi_{\varphi^{\prime} ; L / L_{0}}^{\sigma-1}
$$

because $\Pi_{\varphi ; T / K}^{\varphi}=\Pi_{\varphi ; T / K}$. Moreover, the solution $U=\left(u_{\widetilde{E}}\right) \in U_{\widetilde{\mathbb{X}}\left(L / L_{0}\right)}$, which is unique modulo $U_{\mathbb{X}(L / K)}$, satisfies $\operatorname{Pr}_{L_{0}}(U)=u_{\widetilde{L}_{0}} \in U_{L_{0}}$. In fact, $\operatorname{Pr}_{L_{0}}\left(\Pi_{\varphi^{\prime} ; L / L_{0}}\right)=$ $\pi_{K}$ by Lemma 1.3, whence $\operatorname{Pr}_{L_{0}}\left(\Pi_{\varphi^{\prime} ; L / L_{0}}^{\sigma-1}\right)=\pi_{K}^{\sigma-1}=1_{K}$. Hence, $\operatorname{Pr}_{L_{0}}\left(U^{1-\varphi^{d}}\right)=$ $\operatorname{Pr}_{L_{0}}\left(\Pi_{\varphi^{\prime} ; L / L_{0}}^{\sigma-1}\right)=1_{K}$ yields $u_{\widetilde{L}_{0}}^{1-\varphi^{d}}=1_{K}$, so that $u_{\widetilde{L}_{0}} \in U_{L_{0}}$ because $\widetilde{L}_{0} \cap\left(L_{0}\right)_{\varphi^{\prime}}=L_{0}$. Now, it follows that $\operatorname{Pr}_{L_{0}}(U)=u_{\widetilde{L}_{0}} \in U_{L_{0}}$. Thus, $U=\left(u_{\widetilde{E}}\right)$ belongs to $U_{\widetilde{\mathbb{X}}(L / K)}^{\diamond}$, by Definition 2.1 
Remark 2.3. We can reformulate the definition of the generalized arrow

$$
\phi_{L / K}^{(\varphi)}: \operatorname{Gal}(L / K) \rightarrow K^{\times} / N_{L_{0} / K} L_{0}^{\times} \times U_{\widetilde{\mathbb{X}}(L / K)}^{\diamond} / U_{\mathbb{X}(L / K)}
$$

for the extension $L / K$ as follows:

$$
\phi_{L / K}^{(\varphi)}(\sigma)=\left(\pi_{K}^{m} \cdot N_{L_{0} / K} L_{0}^{\times}, \phi_{L / L_{0}}^{\left(\varphi^{\prime}\right)}\left(\varphi^{-m} \sigma\right)\right)
$$

for every $\sigma \in \operatorname{Gal}(L / K)$, where $\left.\sigma\right|_{L_{0}}=\varphi^{m}$ for some $0 \leq m \in \mathbb{Z}$.

A natural continuous action of $\operatorname{Gal}(L / K)$ on the topological group $K^{\times} / N_{L_{0} / K} L_{0}^{\times} \times$ $U_{\widetilde{\mathbb{X}}(L / K)}^{\diamond} / U_{\mathbb{X}(L / K)}$ is defined by Abelian local class field theory on the first component and by formulas (5.5) and (5.7) in [8] on the second component:

$$
(\bar{a}, \bar{U})^{\sigma}=\left(\bar{a}^{\varphi^{m}}, \bar{U}^{\varphi^{-m} \sigma}\right)=\left(\bar{a}, \bar{U}^{\varphi^{-m} \sigma}\right)
$$

for every $\sigma \in \operatorname{Gal}(L / K)$, where $\left.\sigma\right|_{L_{0}}=\varphi^{m}$ for some $0 \leq m \in \mathbb{Z}$, and for every $a \in K^{\times}$ with $\bar{a}=a \cdot N_{L_{0} / K} L_{0}^{\times}$and every $U \in U_{\widetilde{\mathbb{X}}(L / K)}^{\diamond}$ with $\bar{U}=U \cdot U_{\mathbb{X}(L / K)}$. Below we shall always view $K^{\times} / N_{L_{0} / K} L_{0}^{\times} \times U_{\widetilde{\mathbb{X}}(L / K)}^{\diamond} / U_{\mathbb{X}(L / K)}$ as a topological $\mathrm{Gal}(L / K)$-module.

Theorem 2.4. Let $L / K$ be an infinite APF Galois subextension of $K_{\varphi^{d}} / K$ with residueclass degree $d$. Then the generalized arrow

$$
\phi_{L / K}^{(\varphi)}: \operatorname{Gal}(L / K) \rightarrow K^{\times} / N_{L_{0} / K} L_{0}^{\times} \times U_{\widetilde{\mathbb{X}}(L / K)}^{\diamond} / U_{\mathbb{X}(L / K)}
$$

defined for the extension $L / K$ is an injection, and for every $\sigma, \tau \in \operatorname{Gal}(L / K)$, the cocycle condition

$$
\phi_{L / K}^{(\varphi)}(\sigma \tau)=\phi_{L / K}^{(\varphi)}(\sigma) \phi_{L / K}^{(\varphi)}(\tau)^{\sigma}
$$

is satisfied.

Proof. The injectivity of the arrow (2.3) defined by (2.4) is clear from the canonical topological isomorphism (2.2) combined with Abelian local class field theory and Theorem 5.6 of Fesenko in [8]. More precisely, let $\phi_{L / K}^{(\varphi)}(\sigma)=\left(\pi_{K}^{m},\left(u_{\widetilde{E}}\right)\right)$, where $d \mid m$ and $\left(u_{\widetilde{E}}\right) \in U_{\mathbb{X}\left(L / L_{0}\right)}=U_{\mathbb{X}(L / K)}$. Since $d \mid m$, the action of $\sigma$ is trivial on $L_{0}$. Since $\left(u_{\widetilde{E}}\right)^{\varphi^{d}-1}=\left(1_{\widetilde{E}}\right)=\Pi_{\varphi^{\prime} ; L / L_{0}}^{\sigma-1}, \sigma$ acts trivially on the prime elements of finite subextensions between $L_{0}$ and $L$. Thus, $\sigma$ is the identity element of $\operatorname{Gal}\left(L / L_{0}\right)$. Now, for $\sigma, \tau \in \operatorname{Gal}(L / K)$, with $\left.\sigma\right|_{L_{0}}=\varphi^{m}$ and $\left.\tau\right|_{L_{0}}=\varphi^{n}$ for some $0 \leq m, n \in \mathbb{Z}$, we can use the alternative definition of the generalized arrow $\phi_{L / K}^{(\varphi)}$, introduced in Remark 2.3, to show that

$$
\begin{aligned}
\boldsymbol{\phi}_{L / K}^{(\varphi)}(\sigma \tau) & =\left(\pi_{K}^{m+n} \cdot N_{L_{0} / K} L_{0}^{\times}, \phi_{L / L_{0}}^{\left(\varphi^{\prime}\right)}\left(\varphi^{-(m+n)} \sigma \tau\right)\right) \\
& =\left(\left(\pi_{K}^{m} \cdot N_{L_{0} / K} L_{0}^{\times}\right)\left(\pi_{K}^{n} \cdot N_{L_{0} / K} L_{0}^{\times}\right), \phi_{L / L_{0}}^{\left(\varphi^{\prime}\right)}\left(\varphi^{-m} \sigma\right) \phi_{L / L_{0}}^{\left(\varphi^{\prime}\right)}\left(\varphi^{-n} \tau\right)^{\varphi^{-m} \sigma}\right) \\
& =\left(\pi_{K}^{m} \cdot N_{L_{0} / K} L_{0}^{\times}, \phi_{L / L_{0}}^{\left(\varphi^{\prime}\right)}\left(\varphi^{-m} \sigma\right)\right)\left(\pi_{K}^{n} \cdot N_{L_{0} / K} L_{0}^{\times}, \phi_{L / L_{0}}^{\left(\varphi^{\prime}\right)}\left(\varphi^{-n} \tau\right)^{\varphi^{-m} \sigma}\right) \\
& =\phi_{L / K}^{(\varphi)}(\sigma) \phi_{L / K}^{(\varphi)}(\tau)^{\sigma}
\end{aligned}
$$

by [8, Theorem 5.6] and by the definition of the action of $\sigma \in \operatorname{Gal}(L / K)$ on $\phi_{L / K}^{(\varphi)}(\tau) \in$ $K^{\times} / N_{L_{0} / K} L_{0}^{\times} \times U_{\widetilde{\mathbb{X}}(L / K)}^{\diamond} / U_{\mathbb{X}(L / K)}$, defined by (2.7).

Now, we immediately arrive at the following result. 
Corollary 2.5. Let a law of composition $*$ be defined on $\operatorname{im}\left(\phi_{L / K}^{(\varphi)}\right)$ by

$$
(\bar{a}, \bar{U}) *(\bar{b}, \bar{V})=\left(\bar{a} \cdot \bar{b}, \bar{U} \cdot \bar{V}^{\left(\phi_{L / L_{0}}^{\left(\varphi^{\prime}\right)}\right)^{-1}(\bar{U})}\right)
$$

for $(\bar{a}, \bar{U}),(\bar{b}, \bar{V}) \in \operatorname{im}\left(\phi_{L / K}^{(\varphi)}\right)$ with $\bar{a}=a \cdot N_{L_{0} / K} L_{0}^{\times}, \bar{b}=b \cdot N_{L_{0} / K} L_{0}^{\times} \in K^{\times} / N_{L_{0} / K} L_{0}^{\times}$, and $a, b \in K^{\times}$, and for $\bar{U}=U \cdot U_{\mathbb{X}(L / K)}$ and $\bar{V}=V \cdot U_{\mathbb{X}(L / K)} \in U_{\widetilde{\mathbb{X}}(L / K)}^{\diamond} / U_{\mathbb{X}(L / K)}$ with $U$, $V \in U_{\widetilde{\mathbb{X}}(L / K)}^{\diamond}$. Then $\operatorname{im}\left(\boldsymbol{\phi}_{L / K}^{(\varphi)}\right)$ is a topological group under $*$, and the map $\boldsymbol{\phi}_{L / K}^{(\varphi)}$ induces an isomorphism of topological groups

$$
\phi_{L / K}^{(\varphi)}: \operatorname{Gal}(L / K) \stackrel{\sim}{\longrightarrow} \operatorname{im}\left(\phi_{L / K}^{(\varphi)}\right),
$$

where the topological group structure on $\operatorname{im}\left(\phi_{L / K}^{(\varphi)}\right)$ is defined with respect to the binary operation $*$ defined by (2.9)).

Recall that, for any infinite APF Galois extension $L / K$ and every $-1 \leq u \in \mathbb{R}$, the $u$ th higher ramification subgroup $\operatorname{Gal}(L / K)_{u}$ of $\operatorname{Gal}(L / K)$ in the lower numbering is defined by

$$
\operatorname{Gal}(L / K)_{u}=\operatorname{Gal}(L / K)^{\varphi_{L / K}(u)},
$$

where $-1 \leq \varphi_{L / K}(u) \in \mathbb{R}$ is the number defined by formula (3.1) in [8], and that, as usual, the $\varphi_{L / K}(u)$ th higher ramification subgroup $\operatorname{Gal}(L / K)^{\varphi_{L / K}(u)}$ of $\operatorname{Gal}(L / K)$ in the upper numbering is defined to be the projective limit

$$
\operatorname{Gal}(L / K)^{\varphi_{L / K}(u)}=\lim _{K \subseteq \text { 莐CL }} \operatorname{Gal}(F / K)^{\varphi_{L / K}(u)}
$$

see [8, (2.1) and (2.2)]. Now, let $E / K$ be a Galois subextension of $L / K$. Then, for any chain of field extensions $\overbrace{\underbrace{K \subseteq F}_{\text {finite Gal. }} \subseteq F^{\prime}}^{\text {finite Gal. }} \subset L$, the square

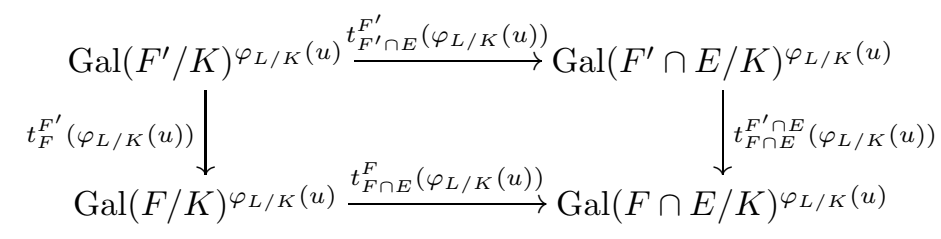

is commutative. Thus, passing to the projective limits, we see that there exists a continuous group homomorphism

$$
t_{E}^{L}\left(\varphi_{L / K}(u)\right)=\lim _{K \subseteq ્(F \subset L} t_{F \cap E}^{F}\left(\varphi_{L / K}(u)\right): \operatorname{Gal}(L / K)^{\varphi_{L / K}(u)} \rightarrow \operatorname{Gal}(E / K)^{\varphi_{L / K}(u)}
$$

which is essentially the restriction morphism from $L$ to $E$. This morphism is a surjection, because the objects in the respective projective systems are compact and Hausdorff. 
Furthermore, the square

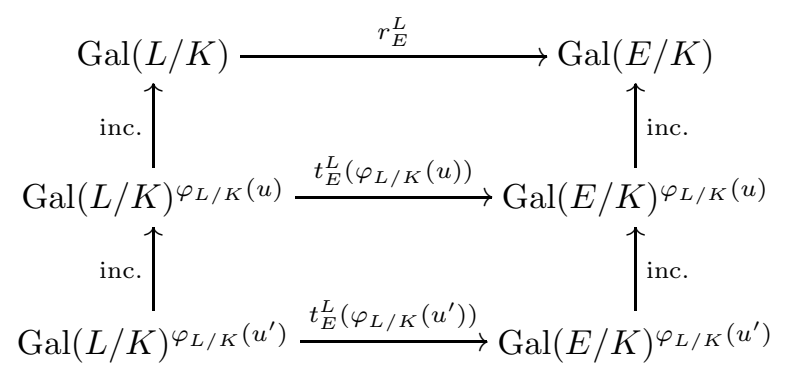

is commutative for every pair $u, u^{\prime} \in \mathbb{R}_{\geq-1}$ satisfying $u \leq u^{\prime}$. Here, the arrow $r_{E}^{L}$ : $\operatorname{Gal}(L / K) \rightarrow \operatorname{Gal}(E / K)$ is the restriction map. Therefore, we arrive at the following result.

Lemma 2.6. For $0 \leq u \in \mathbb{R}$, the topological isomorphism defined by (2.1) and (2.2) induces a topological isomorphism

$$
\operatorname{Gal}(L / K)_{u} \simeq \underbrace{\operatorname{Gal}\left(L_{0} / K\right)^{\varphi_{L / K}(u)}}_{\left\langle\operatorname{id}_{L_{0}}\right\rangle} \times \operatorname{Gal}\left(L / L_{0}\right)^{\varphi_{L / K}(u)}
$$

defined by

$$
\sigma \mapsto\left(t_{L_{0}}^{L}\left(\varphi_{L / K}(u)\right)(\sigma), \varphi^{-m} \sigma\right)=\left(\operatorname{id}_{L_{0}}, \sigma\right)
$$

for every $\sigma \in \operatorname{Gal}(L / K)_{u}$ with $\left.\sigma\right|_{L_{0}}=t_{L_{0}}^{L}\left(\varphi_{L / K}(u)\right)(\sigma)=\left.\varphi^{m}\right|_{L_{0}}$ for some $0 \leq m \in \mathbb{Z}$ satisfying $d \mid m$.

Proof. Note that, for $0 \leq u \in \mathbb{R}, \operatorname{Gal}\left(L_{0} / K\right)^{\varphi_{L / K}(u)}$ is the trivial group $\left\langle\operatorname{id}_{L_{0}}\right\rangle$, because $L_{0} / K$ is a finite unramified extension. Thus, for $\sigma \in \operatorname{Gal}(L / K)_{u}$, by the commutativity of the diagram (2.13) we have

$$
t_{L_{0}}^{L}\left(\varphi_{L / K}(u)\right)(\sigma)=\left.\sigma\right|_{L_{0}}=\operatorname{id}_{L_{0}}
$$

and in return

$$
\sigma \mapsto\left(\operatorname{id}_{L_{0}}, \varphi^{-m} \sigma\right)=\left(\mathrm{id}_{L_{0}}, \sigma\right)
$$

where $\left.\sigma\right|_{L_{0}}=\operatorname{id}_{L_{0}}=\left.\varphi^{m}\right|_{L_{0}}$ for some $0 \leq m \in \mathbb{Z}$ satisfying $d \mid m$. Since $L$ is fixed by $\varphi^{d}$, we have $\varphi^{-m} \sigma=\sigma$, so that $\left(\operatorname{id}_{L_{0}}, \varphi^{-m} \sigma\right)=\left(\operatorname{id}_{L_{0}}, \sigma\right)$. Now, the injectivity of the morphism (2.14), defined by (2.15), is clear from the commutative square (2.13) and from the injectivity of the arrow defined by (2.2). Thus, it suffices to prove that this morphism is a surjection, which follows from the triviality of $\operatorname{Gal}\left(L_{0} / K\right)^{\varphi_{L / K}(u)}$ for $0 \leq u \in \mathbb{R}$, and from the fact that $\operatorname{Gal}(L / K)_{u}=\operatorname{Gal}\left(L / L_{0}\right)^{\varphi_{L / K}(u)}$.

Now, $L / L_{0}$ is an APF Galois subextension of $L / K$ by part (i) of Lemma 3.3 in 8 . Let $\varphi_{L / L_{0}}: \mathbb{R}_{\geq-1} \rightarrow \mathbb{R}_{\geq-1}$ be the Hasse-Herbrand function corresponding to the APF extension $L / L_{0}$ defined by relation (3.1) in $[8$, which is piecewise-linear and continuous. So, there exists a unique number $w=w(u, L / K) \in \mathbb{R}_{\geq-1}$ depending on $u$, satisfying $\varphi_{L / K}(u)=\varphi_{L / L_{0}}(w)$, and such that

$$
\operatorname{Gal}\left(L / L_{0}\right)^{\varphi_{L / K}(u)}=\operatorname{Gal}\left(L / L_{0}\right)^{\varphi_{L / L_{0}}(w)}=\operatorname{Gal}\left(L / L_{0}\right)_{w} .
$$

Thus, Lemma 2.6 can be reformulated as follows. The topological isomorphism defined by (2.1) and (2.2) induces a topological isomorphism

$$
\operatorname{Gal}(L / K)_{u} \simeq\left\langle\operatorname{id}_{L_{0}}\right\rangle \times \operatorname{Gal}\left(L / L_{0}\right)_{w(u, L / K)}
$$

for every $0 \leq u \in \mathbb{R}$. 
For each $0 \leq i \in \mathbb{R}$, we consider the $i$ th higher unit group $U_{\widetilde{\mathbb{X}}(L / K)}^{i}$ of the field $\widetilde{\mathbb{X}}(L / K)$ and introduce the group

$$
\left(U_{\widetilde{\mathbb{X}}(L / K)}^{\diamond}\right)^{i}=U_{\widetilde{\mathbb{X}}(L / K)}^{\diamond} \cap U_{\widetilde{\mathbb{X}}(L / K)}^{i}
$$

Now, the Fesenko ramification theorem, stated as Theorem 5.8 in [8], has the following generalization for the generalized arrow $\phi_{L / K}^{(\varphi)}$ corresponding to the extension $L / K$ that is an infinite APF Galois subextension of $K_{\varphi^{d}} / K$ with residue-class degree $\left[\kappa_{L}: \kappa_{K}\right]=d$.

Theorem 2.7 (Ramification theorem). For $0 \leq u \in \mathbb{R}$, let $\operatorname{Gal}(L / K)_{u}$ denote the uth higher ramification subgroup in the lower numbering of the Galois group $\operatorname{Gal}(L / K)$ corresponding to the infinite APF Galois subextension $L / K$ of $K_{\varphi^{d}} / K$ with residue-class degree $\left[\kappa_{L}: \kappa_{K}\right]=d$. Then, for $0 \leq n \in \mathbb{Z}$, we have the inclusion

$$
\begin{aligned}
& \phi_{L / K}^{(\varphi)}\left(\operatorname{Gal}(L / K)_{\psi_{L / K} \circ \varphi_{L / L_{0}}(n)}-\operatorname{Gal}(L / K)_{\psi_{L / K} \circ \varphi_{L / L_{0}}(n+1)}\right) \\
& \subseteq\left\langle 1_{K^{\times} / N_{L_{0} / K} L_{0}^{\times}}\right\rangle \\
& \quad \times\left(\left(U_{\widetilde{\mathbb{X}}(L / K)}^{\diamond}\right)^{n} U_{\mathbb{X}(L / K)} / U_{\mathbb{X}(L / K)}-\left(U_{\widetilde{\mathbb{X}}(L / K)}^{\diamond}\right)^{n+1} U_{\mathbb{X}(L / K)} / U_{\mathbb{X}(L / K)}\right) .
\end{aligned}
$$

Proof. We start with the following general observation. Let $0 \leq u \in \mathbb{R}$. Let $\tau \in$ $\operatorname{Gal}(L / K)_{u}=\operatorname{Gal}\left(L / L_{0}\right)^{\varphi_{L / K}(u)}$. Then, by the definition of the generalized arrow $\boldsymbol{\phi}_{L / K}^{(\varphi)}$ reformulated as in Remark 2.3

$$
\boldsymbol{\phi}_{L / K}^{(\varphi)}(\tau)=\left(\pi_{K}^{m} \cdot N_{L_{0} / K} L_{0}^{\times}, \phi_{L / L_{0}}^{\left(\varphi^{\prime}\right)}\left(\varphi^{-m} \tau\right)\right),
$$

where $\left.\tau\right|_{L_{0}}=\left.\varphi^{m}\right|_{L_{0}}$ for some $0 \leq m \in \mathbb{Z}$ satisfying $d \mid m$, because $\tau \in \operatorname{Gal}(L / K)_{u}$ and $\left.\tau\right|_{L_{0}}=t_{L_{0}}^{L}\left(\varphi_{L / K}(u)\right)(\tau) \in \operatorname{Gal}\left(L_{0} / K\right)^{\varphi_{L / K}(u)}=\left\langle\operatorname{id}_{L_{0}}\right\rangle$. Thus,

$$
\boldsymbol{\phi}_{L / K}^{(\varphi)}(\tau)=\left(1_{K^{\times} / N_{L_{0} / K} L_{0}^{\times}}, \phi_{L / L_{0}}^{\left(\varphi^{\prime}\right)}\left(\varphi^{-m} \tau\right)\right),
$$

as $m=d m^{\prime}$ and thereby $\pi_{K}^{d m^{\prime}} N_{L_{0} / K} L_{0}^{\times}=N_{L_{0} / K} L_{0}^{\times}=1_{K \times / N_{L_{0} / K} L_{0}^{\times}}$since $N_{L_{0} / K} \pi_{K}^{m^{\prime}}=$ $\pi_{K}^{m}$. Therefore, we have

$$
\phi_{L / K}^{(\varphi)}(\tau)=\left(1_{K \times / N_{L_{0} / K} L_{0}^{\times}}, \phi_{L / L_{0}}^{\left(\varphi^{\prime}\right)}(\tau)\right)
$$

Indeed, $\varphi^{-m} \tau=\tau$ in $\operatorname{Gal}\left(L / L_{0}\right)$ because $d \mid m$ and $L \subset K_{\varphi^{d}}$.

Now, to prove the theorem, we put $u=\psi_{L / K} \circ \varphi_{L / L_{0}}(n)$ and $u^{\prime}=\psi_{L / K} \circ \varphi_{L / L_{0}}(n+1)$. Then, for any $\tau \in \operatorname{Gal}(L / K)_{u}-\operatorname{Gal}(L / K)_{u^{\prime}}$, the Fesenko ramification theorem (see [8, Theorem 5.8]) shows that the second coordinate of $\phi_{L / K}^{(\varphi)}(\tau)$ satisfies

$$
\phi_{L / L_{0}}^{\left(\varphi^{\prime}\right)}(\tau) \in\left(U_{\widetilde{\mathbb{X}}(L / K)}^{\diamond}\right)^{n} U_{\mathbb{X}(L / K)} / U_{\mathbb{X}(L / K)}-\left(U_{\widetilde{\mathbb{X}}(L / K)}^{\diamond}\right)^{n+1} U_{\mathbb{X}(L / K)} / U_{\mathbb{X}(L / K)},
$$

because

$$
\operatorname{Gal}(L / K)_{u}=\operatorname{Gal}\left(L / L_{0}\right)^{\varphi_{L / K}(u)}=\operatorname{Gal}\left(L / L_{0}\right)^{\varphi_{L / L_{0}}(n)}=\operatorname{Gal}\left(L / L_{0}\right)_{n}
$$

and likewise

$$
\operatorname{Gal}(L / K)_{u^{\prime}}=\operatorname{Gal}\left(L / L_{0}\right)^{\varphi_{L / K}\left(u^{\prime}\right)}=\operatorname{Gal}\left(L / L_{0}\right)^{\varphi_{L / L_{0}}(n+1)}=\operatorname{Gal}\left(L / L_{0}\right)_{n+1},
$$

which completes the proof. 
Now, let $M / K$ be an infinite Galois subextension of $L / K$. Thus, by [8, Lemma 3.3], $M$ is an APF Galois extension over $K$. We further assume that the residue-class degree $\left[\kappa_{M}: \kappa_{K}\right]$ is equal to $d^{\prime}$ and $K \subset M \subset K_{\varphi^{d^{\prime}}}$ for some $d^{\prime} \mid d$. Let

$$
\phi_{M / K}^{(\varphi)}: \operatorname{Gal}(M / K) \rightarrow K^{\times} / N_{M_{0} / K} M_{0}^{\times} \times U_{\widetilde{\mathbb{X}}(M / K)}^{\ominus} / U_{\mathbb{X}(M / K)}
$$

be the corresponding generalized arrow defined for the extension $M / K$. Here, $M_{0}=$ $M \cap K^{n r}=K_{d^{\prime}}^{n r}$.

Let

$$
K \subset L_{o}=E_{o} \subset E_{1} \subset \cdots \subset E_{i} \subset \cdots \subset L
$$

be an ascending chain satisfying $L=\bigcup_{0 \leq i \in \mathbb{Z}} E_{i}$ and $\left[E_{i+1}: E_{i}\right]<\infty$ for every $0 \leq i \in \mathbb{Z}$. Then

$$
K \subset M_{o}=E_{o} \cap M \subseteq E_{1} \cap M \subseteq \cdots \subseteq E_{i} \cap M \subseteq \cdots \subset M
$$

is an ascending chain of field extensions satisfying $M=\bigcup_{0 \leq i \in \mathbb{Z}}\left(E_{i} \cap M\right)$ and also $\left[E_{i+1} \cap M: E_{i} \cap M\right]<\infty$ for every $0 \leq i \in \mathbb{Z}$. Thus, we construct $\mathbb{X}(M / K)$ by the sequence $\left(E_{i} \cap M\right)_{0 \leq i \in \mathbb{Z}}$ and $\widetilde{\mathbb{X}}(M / K)$ by the sequence $\left(\widetilde{E_{i} \cap M}\right)_{0 \leq i \in \mathbb{Z}}$. Observe that $E_{i} \cap M \neq E_{i}$ for every $0 \leq i \in \mathbb{Z}$. Furthermore, the commutative square

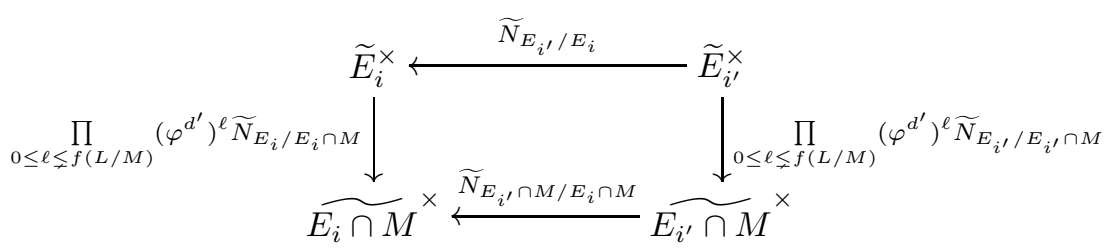

for every pair $0 \leq i, i^{\prime} \in \mathbb{Z}$ satisfying $i \leq i^{\prime}$, induces the group homomorphism

$$
\widetilde{\mathcal{N}}_{L / M}=\lim _{0 \leq i \in \mathbb{Z}}\left(\prod_{0 \leq \ell \leq f(L / M)}\left(\varphi^{d^{\prime}}\right)^{\ell} \widetilde{N}_{E_{i} / E_{i} \cap M}\right): \widetilde{\mathbb{X}}(L / K)^{\times} \rightarrow \widetilde{\mathbb{X}}(M / K)^{\times}
$$

defined by

$$
\widetilde{\mathcal{N}}_{L / M}\left(\left(\alpha_{\widetilde{E}_{i}}\right)_{0 \leq i \in \mathbb{Z}}\right)=\left(\prod_{0 \leq \ell \leq f(L / M)}\left(\varphi^{d^{\prime}}\right)^{\ell} \widetilde{N}_{E_{i} / E_{i} \cap M}\left(\alpha_{\widetilde{E}_{i}}\right)\right)_{0 \leq i \in \mathbb{Z}},
$$

for every $\left(\alpha_{\widetilde{E}_{i}}\right)_{0 \leq i \in \mathbb{Z}} \in \widetilde{\mathbb{X}}(L / K)^{\times}$.

Remark 2.8. The group homomorphism

$$
\widetilde{\mathcal{N}}_{L / M}: \widetilde{\mathbb{X}}(L / K)^{\times} \rightarrow \widetilde{\mathbb{X}}(M / K)^{\times}
$$

defined by (2.17) and (2.18) does not depend on the choice of the ascending chain

$$
K \subset L_{o}=E_{o} \subset E_{1} \subset \cdots \subset E_{i} \subset \cdots \subset L
$$

satisfying $L=\bigcup_{0 \leq i \in \mathbb{Z}} E_{i}$ and $\left[E_{i+1}: E_{i}\right]<\infty$ for every $0 \leq i \in \mathbb{Z}$.

Remark 2.9. For $0 \leq i \in \mathbb{Z}$, let $E_{i, 0}^{\left(E_{i} \cap M\right)}=E_{i} \cap\left(E_{i} \cap M\right)^{n r}$ be the maximal unramified extension of $E_{i} \cap M$ inside $E_{i}$. To simplify the notation, we put $E_{i, 0}=E_{i} \cap\left(E_{i} \cap M\right)^{n r}$. Then the Galois group $\operatorname{Gal}\left(E_{i, 0} / E_{i} \cap M\right)$ is cyclic of order $f(L / M)=\frac{d}{d^{\prime}}$ and is generated by $\varphi^{d^{\prime}}$. Thus, for $\alpha \in E_{i}$,

$$
N_{E_{i} / E_{i} \cap M}(\alpha)=\widetilde{N}_{E_{i} / E_{i} \cap M}(\alpha)^{1+\varphi^{d^{\prime}}+\cdots+\varphi^{d^{\prime}(f(L / M)-1)}} .
$$

The basic properties of this group homomorphism are listed below.

(i) If $U=\left(u_{\widetilde{E}_{i}}\right)_{0 \leq i \in \mathbb{Z}} \in U_{\widetilde{\mathbb{X}}(L / K)}$, then $\widetilde{\mathcal{N}}_{L / M}(U) \in U_{\widetilde{\mathbb{X}}(M / K)}$. 
Proof. Indeed, using the definition of the valuation $\nu_{\widetilde{\mathbb{X}}(M / K)}$ of $\widetilde{\mathbb{X}}(M / K)$ and the definition of the valuation $\nu_{\widetilde{\mathbb{X}}(L / K)}$ of $\widetilde{\mathbb{X}}(L / K)$, we obtain

$$
\begin{aligned}
\nu_{\widetilde{\mathbb{X}}(M / K)}\left(\widetilde{\mathcal{N}}_{L / M}(U)\right) & \left.=\nu_{\widetilde{\mathbb{X}}(M / K)}\left(\prod_{0 \leq \ell \leq f(L / M)}\left(\varphi^{d^{\prime}}\right)^{\ell} \widetilde{N}_{E_{i} / E_{i} \cap M}\left(u_{\widetilde{E}_{i}}\right)\right)_{0 \leq i \in \mathbb{Z}}\right) \\
& =\nu_{\widetilde{K}}\left(\prod_{0 \leq \ell \leq f(L / M)}\left(\varphi^{d^{\prime}}\right)^{\ell}\left(u_{\widetilde{K}}\right)\right)=\sum_{0 \leq \ell \leq f(L / M)} \nu_{\widetilde{K}}\left(\left(\varphi^{d^{\prime}}\right)^{\ell}\left(u_{\widetilde{K}}\right)\right) \\
& =\sum_{0 \leq \ell \leq f(L / M)} \nu_{\widetilde{K}}\left(u_{\widetilde{K}}\right)=0,
\end{aligned}
$$

because $\nu_{\widetilde{K}}\left(\left(\varphi^{d^{\prime}}\right)^{\ell}\left(u_{\widetilde{K}}\right)\right)=\nu_{\widetilde{K}}\left(u_{\widetilde{K}}\right)$ for $\ell=1, \ldots, f(L / M)-1$, and

$$
\nu_{\widetilde{\mathbb{X}}(L / K)}(U)=\nu_{\widetilde{K}}\left(u_{\widetilde{K}}\right)=0,
$$

because $U \in U_{\widetilde{\mathbb{X}}(L / K)}$.

(ii) If $U=\left(u_{\widetilde{E}_{i}}\right)_{0 \leq i \in \mathbb{Z}} \in U_{\widetilde{\mathbb{X}}(L / K)}^{\diamond}$, then $\widetilde{\mathcal{N}}_{L / M}(U) \in U_{\widetilde{\mathbb{X}}(M / K)}^{\diamond}$.

Proof. Note that $\widetilde{L}_{0}=\widetilde{K}$ and $\widetilde{M}_{0}=\widetilde{K}$. Now, the claim follows from the observation that

and

$$
\operatorname{Pr}_{\widetilde{K}}(U)=u_{\widetilde{K}} \in U_{L_{0}}
$$

$$
\begin{aligned}
\operatorname{Pr}_{\widetilde{K}}\left(\widetilde{\mathcal{N}}_{L / M}(U)\right) & =\prod_{0 \leq \ell \leq f(L / M)}\left(\varphi^{d^{\prime}}\right)^{\ell} \widetilde{N}_{E_{o} / E_{o} \cap M}\left(u_{\widetilde{E}_{o}}\right) \\
& =\prod_{0 \leq \ell \leq f(L / M)}\left(\varphi^{d^{\prime}}\right)^{\ell} u_{\widetilde{K}}=N_{E_{o} / E_{o} \cap M}\left(u_{\widetilde{K}}\right) \in U_{M_{0}} .
\end{aligned}
$$

(iii) If $U=\left(u_{E_{i}}\right)_{0 \leq i \in \mathbb{Z}} \in U_{\mathbb{X}(L / K)}$, then $\widetilde{\mathcal{N}}_{L / M}(U) \in U_{\mathbb{X}(M / K)}$.

Proof. This follows from the definition (2.18) of the homomorphism (2.17), combined with the fact that

$$
\widetilde{N}_{E_{i} / E_{i} \cap M}\left(u_{E_{i}}\right)^{1+\varphi^{d^{\prime}}+\cdots+\varphi^{d^{\prime}(f(L / M)-1)}}=N_{E_{i} / E_{i} \cap M}\left(u_{E_{i}}\right)
$$

for every $u_{E_{i}} \in U_{E_{i}}$ and every $0 \leq i \in \mathbb{Z}$, by Remark 2.9 .

Observe that $\widetilde{N}_{E_{i} / E_{i-1}}\left(\alpha^{1+\varphi^{d^{\prime}}+\cdots+\varphi^{d^{\prime}(f-1)}}\right)=\widetilde{N}_{E_{i} / E_{i-1}}(\alpha)^{1+\varphi^{d^{\prime}}+\cdots+\varphi^{d^{\prime}(f-1)}}$ for any $\alpha \in \widetilde{E}_{i}$ with $1 \leq i \in \mathbb{Z}$, where $f=f(L / M)$. Thus, we can define a homomorphism

$$
\langle\varphi\rangle_{L / M}: \widetilde{\mathbb{X}}\left(L / L_{0}\right)^{\times} \rightarrow \widetilde{\mathbb{X}}\left(L / L_{0}\right)^{\times}
$$

by the rule

$$
\langle\varphi\rangle_{L / M}:\left(\alpha_{\widetilde{E}_{i}}\right)_{0 \leq i \in \mathbb{Z}} \mapsto\left(\alpha_{\widetilde{E}_{i}}^{1+\varphi^{d^{\prime}}+\cdots+\varphi^{d^{\prime}(f-1)}}\right)_{0 \leq i \in \mathbb{Z}}
$$

for every $\left(\alpha_{\widetilde{E}_{i}}\right)_{0 \leq i \in \mathbb{Z}} \in \widetilde{\mathbb{X}}\left(L / L_{0}\right)^{\times}$. The basic properties of this group homomorphism are listed below.

(i) $\langle\varphi\rangle_{L / M}\left(U_{\widetilde{\mathbb{X}}\left(L / L_{0}\right)}\right) \subseteq U_{\widetilde{\mathbb{X}}\left(L / L_{0}\right)}$. 
Proof. Indeed, the definition of the valuation $\nu_{\widetilde{\mathbb{X}}\left(L / L_{0}\right)}$ of $\widetilde{\mathbb{X}}\left(L / L_{0}\right)$ shows that

$$
\begin{aligned}
\nu_{\widetilde{\mathbb{X}}\left(L / L_{0}\right)}\left(\langle\varphi\rangle_{L / M}(U)\right) & =\nu_{\widetilde{\mathbb{X}}\left(L / L_{0}\right)}\left(u_{\widetilde{E}}^{1+\varphi^{d^{\prime}}+\cdots+\varphi^{d^{\prime}(f(L / M)-1)}}\right)_{0 \leq i \in \mathbb{Z}} \\
& =\nu_{\widetilde{K}}\left(u_{\widetilde{K}}^{1+\varphi^{d^{\prime}}+\cdots+\varphi^{d^{\prime}(f(L / M)-1)}}\right)=\sum_{0 \leq \ell \leq f(L / M)} \nu_{\widetilde{K}}\left(\left(\varphi^{d^{\prime}}\right)^{\ell}\left(u_{\widetilde{K}}\right)\right) \\
& =\sum_{0 \leq \ell \leq f(L / M)} \nu_{\widetilde{K}}\left(u_{\widetilde{K}}\right)=0,
\end{aligned}
$$

because $\nu_{\widetilde{K}}\left(\left(\varphi^{d^{\prime}}\right)^{\ell}\left(u_{\widetilde{K}}\right)\right)=\nu_{\widetilde{K}}\left(u_{\widetilde{K}}\right)$ for $\ell=1, \ldots, f(L / M)-1$, and

$$
\nu_{\widetilde{\mathbb{X}}(L / K)}(U)=\nu_{\widetilde{K}}\left(u_{\widetilde{K}}\right)=0
$$

because $U \in U_{\widetilde{\mathbb{X}}(L / K)}$.

(ii) $\langle\varphi\rangle_{L / M}\left(U_{\widetilde{\mathbb{X}}\left(L / L_{0}\right)}^{\diamond}\right) \subseteq U_{\widetilde{\mathbb{X}}\left(L / L_{0}\right)}^{\diamond}$.

Proof. Note that $\widetilde{L}_{0}=\widetilde{K}$. Now, the claim follows from the observation that

$$
\operatorname{Pr}_{\widetilde{K}}(U)=u_{\widetilde{K}} \in U_{L_{0}}
$$

and

$$
\begin{aligned}
\operatorname{Pr}_{\widetilde{K}}\left(\langle\varphi\rangle_{L / M}(U)\right) & =u_{\widetilde{K}}^{1+\varphi^{d^{\prime}}+\cdots+\varphi^{d^{\prime}(f(L / M)-1)}} \\
& =\prod_{0 \leq \ell \leq f(L / M)}\left(\varphi^{d^{\prime}}\right)^{\ell} u_{\widetilde{K}}=N_{E_{o} / E_{o} \cap M}\left(u_{\widetilde{K}}\right) \in U_{M_{0}} \subseteq U_{L_{0}} .
\end{aligned}
$$

(iii) $\langle\varphi\rangle_{L / M}\left(U_{\mathbb{X}\left(L / L_{0}\right)}\right) \subseteq U_{\mathbb{X}\left(L / L_{0}\right)}$.

Proof. Clearly, for $U=\left(u_{E_{i}}\right)_{0 \leq i \in \mathbb{Z}} \in U_{\mathbb{X}\left(L / L_{o}\right)}$ we have

$$
\langle\varphi\rangle_{L / M}(U)=\left(u_{E_{i}}^{1+\varphi^{d^{\prime}}+\cdots+\varphi^{d^{\prime}(f(L / M)-1)}}\right)_{0 \leq i \in \mathbb{Z}} \in U_{\mathbb{X}\left(L / L_{o}\right)}
$$

because $u_{E_{i}} \in U_{E_{i}}$ for every $0 \leq i \in \mathbb{Z}$.

Thus, there exists a group homomorphism

$$
\tilde{\mathcal{N}}_{L / M} \circ\langle\varphi\rangle_{L / M}: \widetilde{\mathbb{X}}(L / K)^{\times} \rightarrow \widetilde{\mathbb{X}}(M / K)^{\times}
$$

satisfying

(i) $\widetilde{\mathcal{N}}_{L / M} \circ\langle\varphi\rangle_{L / M}\left(U_{\widetilde{\mathbb{X}}(L / K)}\right) \subseteq U_{\widetilde{\mathbb{X}}(M / K)}$;

(ii) $\tilde{\mathcal{N}}_{L / M} \circ\langle\varphi\rangle_{L / M}\left(U_{\widetilde{\mathbb{X}}(L / K)}^{\diamond}\right) \subseteq U_{\widetilde{\mathbb{X}}(M / K)}^{\diamond}$;

(iii) $\widetilde{\mathcal{N}}_{L / M} \circ\langle\varphi\rangle_{L / M}\left(U_{\mathbb{X}(L / K)}\right) \subseteq U_{\mathbb{X}(M / K)}$.

Now, we introduce the Coleman norm map

$$
\widetilde{\mathcal{N}}_{L / M}^{\text {Coleman }}: U_{\widetilde{\mathbb{X}}(L / K)}^{\diamond} / U_{\mathbb{X}(L / K)} \rightarrow U_{\widetilde{\mathbb{X}}(M / K)}^{\diamond} / U_{\mathbb{X}(M / K)}
$$

from $L$ to $M$ by

$$
\widetilde{\mathcal{N}}_{L / M}^{\text {Coleman }}(\bar{U})=\widetilde{\mathcal{N}}_{L / M} \circ\langle\varphi\rangle_{L / M}(U) \cdot U_{\mathbb{X}(M / K)}
$$

for every $U \in U_{\widetilde{\mathbb{X}}(L / K)}^{\diamond}$, where, as before, $\bar{U}$ is the coset $U \cdot U_{\mathbb{X}(L / K)}$ in $U_{\widetilde{\mathbb{X}}(L / K)}^{\diamond} / U_{\mathbb{X}(L / K)}$. 
Lemma 2.10. For an infinite Galois subextension $M / K$ of $L / K$ such that the residueclass degree $\left[\kappa_{M}: \kappa_{K}\right]$ is equal to $d^{\prime}$ and $K \subset M \subset K_{\varphi^{d^{\prime}}}$ for some $d^{\prime} \mid d$, the square

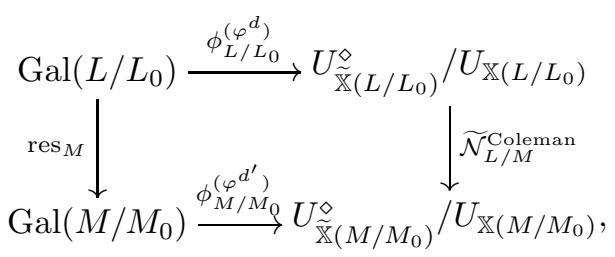

where the right-vertical arrow is the Coleman norm map $\tilde{\mathcal{N}}_{L / M}^{\text {Coleman }}$ from $L$ to $M$ defined by (2.22) and (2.23), is commutative.

Proof. For $\sigma \in \operatorname{Gal}\left(L / L_{0}\right)$, we have $\operatorname{res}_{M}(\sigma)=\left.\sigma\right|_{M} \in \operatorname{Gal}\left(M / M_{0}\right)$, because $L_{0} \cap M=$ $L \cap K^{n r} \cap M=M \cap K^{n r}=M_{0}$. Now, for any $\sigma \in \operatorname{Gal}\left(L / L_{0}\right)$, in accordance with the definition, we can write $\phi_{L / L_{0}}^{\left(\varphi^{d}\right)}(\sigma)=U_{\sigma} \cdot U_{\mathbb{X}\left(L / L_{0}\right)}$, where $U_{\sigma} \in U_{\widetilde{\mathbb{X}}\left(L / L_{0}\right)}^{\diamond}$ satisfies the equation $U_{\sigma}^{1-\varphi^{d}}=\Pi_{\varphi^{d} ; L / L_{0}}^{\sigma-1}$. Thus, to prove the commutativity of the square, it suffices to check that

$$
\widetilde{\mathcal{N}}_{L / M}\left(U_{\sigma}^{1+\varphi^{d^{\prime}}+\cdots+\varphi^{d^{\prime}(f(L / M)-1)}}\right) \equiv U_{\left.\sigma\right|_{M}} \quad\left(\bmod U_{\mathbb{X}\left(M / M_{0}\right)}\right),
$$

where $U_{\left.\sigma\right|_{M}} \in U_{\widetilde{\mathbb{X}}\left(M / M_{0}\right)}^{\diamond}$ satisfies $U_{\left.\sigma\right|_{M}}^{1-{d^{d}}^{d^{\prime}}}=\Pi_{\varphi^{d^{\prime} ; M / M_{0}}}^{\left.\sigma\right|_{M}-1}$. By Remark 2.8, without loss of generality we can fix a basic sequence (cf. [8] )

$$
L_{0}=E_{0} \subset E_{1} \subset \cdots \subset E_{i} \subset \cdots \subset L,
$$

where

(i) $L=\bigcup_{0 \leq i \in \mathbb{Z}} E_{i}$;

(ii) $E_{i} / L_{0}$ is a Galois extension for every $0 \leq i \in \mathbb{Z}$;

(iii) $E_{i+1} / E_{i}$ is cyclic of prime degree $\left[E_{i+1}: E_{i}\right]=p=\operatorname{char}\left(\kappa_{L_{0}}\right)$ for each $1 \leq i \in \mathbb{Z}$;

(iv) $E_{1} / E_{0}$ is cyclic of degree relatively prime to $p$.

Thus, each extension $E_{i} / L_{0}$ is finite and Galois for $0 \leq i \in \mathbb{Z}$. Now, we note that

$$
\widetilde{\mathcal{N}}_{L / M}\left(U_{\sigma}^{1+\varphi^{d^{\prime}}+\cdots+\varphi^{d^{\prime}(f(L / M)-1)}}\right)^{1-\varphi^{d^{\prime}}}=\widetilde{\mathcal{N}}_{L / M}\left(U_{\sigma}\right)^{1-\varphi^{d}}=\widetilde{\mathcal{N}}_{L / M}\left(U_{\sigma}^{1-\varphi^{d}}\right)
$$

Since $U_{\sigma}^{1-\varphi^{d}}=\Pi_{\varphi^{d} ; L / L_{0}}^{\sigma-1}$, setting $U_{\sigma}=\left(u_{\widetilde{E}_{i}}\right)_{0 \leq i \in \mathbb{Z}}$, for $0 \leq i \in \mathbb{Z}$ we obtain

$$
\begin{aligned}
& \widetilde{\mathcal{N}}_{L / M}\left(U_{\sigma}^{1+\varphi^{d^{\prime}}+\cdots+\varphi^{d^{\prime}(f(L / M)-1)}}\right)_{i}^{1-\varphi^{d^{\prime}}} \\
& =\widetilde{\mathcal{N}}_{L / M}\left(\Pi_{\varphi^{d} ; L / L_{0}}^{\sigma-1}\right)_{i}=\widetilde{N}_{E_{i} / E_{i} \cap M}\left(\pi_{E_{i}}^{\sigma-1}\right)^{1+\varphi^{d^{\prime}}+\cdots+\varphi^{d^{\prime}(f(L / M)-1)}} \\
& =N_{E_{i} / E_{i} \cap M}\left(\pi_{E_{i}}^{\sigma-1}\right)=\pi_{E_{i} \cap M}^{\left.\sigma\right|_{M}-1} .
\end{aligned}
$$

It follows that $\tilde{\mathcal{N}}_{L / M} \circ\langle\varphi\rangle_{L / M}\left(U_{\sigma}\right)^{1-\varphi^{d^{\prime}}}=\Pi_{\varphi^{d^{\prime}} ; M / M_{0}}^{\left.\sigma\right|_{M}-1}$, which yields the congruence $\widetilde{\mathcal{N}}_{L / M} \circ\langle\varphi\rangle_{L / M}\left(U_{\sigma}\right) \equiv U_{\left.\sigma\right|_{M}}\left(\bmod U_{\mathbb{X}\left(M / M_{0}\right)}\right)$. This completes the proof.

We arrive at the following theorem.

Theorem 2.11. For an infinite Galois subextension $M / K$ of $L / K$ such that the residueclass degree $\left[\kappa_{M}: \kappa_{K}\right]$ is equal to $d^{\prime}$ and $K \subset M \subset K_{\varphi^{d^{\prime}}}$ for some $d^{\prime} \mid d$, the following 
square is commutative:

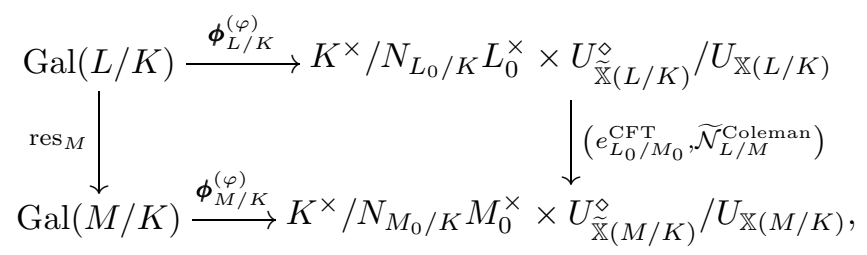

where the right vertical arrow

$$
\begin{aligned}
K^{\times} / N_{L_{0} / K} L_{0}^{\times} & \times U_{\widetilde{\mathbb{X}}(L / K)}^{\diamond} / U_{\mathbb{X}(L / K)} \frac{\left(e_{L_{0} / M_{0}}^{\mathrm{CFT}} \widetilde{\mathcal{N}}_{L / M}^{\text {Coleman }}\right)}{\longrightarrow} K^{\times} / N_{M_{0} / K} M_{0}^{\times} \\
& \times U_{\widetilde{\mathbb{X}}(M / K)}^{\diamond} / U_{\mathbb{X}(M / K)}
\end{aligned}
$$

is defined by the rule

$$
\left(e_{L_{0} / M_{0}}^{\mathrm{CFT}}, \tilde{\mathcal{N}}_{L / M}^{\text {Coleman }}\right):(\bar{a}, \bar{U}) \mapsto\left(e_{L_{0} / M_{0}}^{\mathrm{CFT}}(\bar{a}), \tilde{\mathcal{N}}_{L / M}^{\text {Coleman }}(\bar{U})\right)
$$

for every $(\bar{a}, \bar{U}) \in K^{\times} / N_{L_{0} / K} L_{0}^{\times} \times U_{\widetilde{\mathbb{X}}(L / K)}^{\diamond} / U_{\mathbb{X}(L / K)}$. Here,

$$
e_{L_{0} / M_{0}}^{\mathrm{CFT}}: K^{\times} / N_{L_{0} / K} L_{0}^{\times} \rightarrow K^{\times} / N_{M_{0} / K} M_{0}^{\times}
$$

is the natural inclusion defined via the existence theorem of the local class field theory.

Proof. By the isomorphism defined by (2.1) and (2.2), for any $\sigma \in \operatorname{Gal}(L / K)$ there exists a unique $0 \leq m \in \mathbb{Z}$ such that $\left.\sigma\right|_{L_{0}}=\varphi^{m}$ and $\varphi^{-m} \sigma \in \operatorname{Gal}\left(L / L_{0}\right)$. By the definition, we have

$$
\boldsymbol{\phi}_{L / K}^{(\varphi)}(\sigma)=\left(\pi_{K}^{m} N_{L_{0} / K} L_{0}^{\times}, \phi_{L / L_{0}}^{\left(\varphi^{d}\right)}\left(\varphi^{-m} \sigma\right)\right)
$$

Thus,

$$
\begin{aligned}
& \left(e_{L_{0} / M_{0}}^{\mathrm{CFT}}, \tilde{\mathcal{N}}_{L / M}^{\text {Coleman }}\right)\left(\pi_{K}^{m} N_{L_{0} / K} L_{0}^{\times}, \phi_{L / L_{0}}^{\left(\varphi^{d}\right)}\left(\varphi^{-m} \sigma\right)\right) \\
& \quad=\left(e_{L_{0} / M_{0}}^{\mathrm{CFT}}\left(\pi_{K}^{m} N_{L_{0} / K} L_{0}^{\times}\right), \tilde{\mathcal{N}}_{L / M}^{\text {Coleman }}\left(\phi_{L / L_{0}}^{\left(\varphi^{d}\right)}\left(\varphi^{-m} \sigma\right)\right)\right) \\
& \quad=\left(\pi_{K}^{m} N_{M_{0} / K} M_{0}^{\times}, \phi_{M / M_{0}}^{\left(\varphi^{d^{\prime}}\right)}\left(\left.\varphi^{-m} \sigma\right|_{M}\right)\right)
\end{aligned}
$$

by Lemma 2.10, The existence theorem of the local class field theory yields

$$
e_{L_{0} / M_{0}}^{\mathrm{CFT}}\left(\pi_{K}^{m} N_{L_{0} / K} L_{0}^{\times}\right)=\pi_{K}^{m} N_{M_{0} / K} M_{0}^{\times}=\pi_{K}^{m^{\prime}} N_{M_{0} / K} M_{0}^{\times},
$$

where $0 \leq m^{\prime} \in \mathbb{Z}$ is a unique integer satisfying $\left.\left(\left.\sigma\right|_{M}\right)\right|_{M_{0}}=\left.\sigma\right|_{M_{0}}=\varphi^{m^{\prime}}$ and $\varphi^{-m^{\prime}}\left(\left.\sigma\right|_{M}\right) \in$ $\operatorname{Gal}\left(M / M_{0}\right)$. Hence,

$$
\begin{aligned}
\left(e_{L_{0} / M_{0}}^{\mathrm{CFT}}, \tilde{\mathcal{N}}_{L / M}^{\text {Coleman }}\right)\left(\phi_{L / K}^{(\varphi)}(\sigma)\right)=\left(\pi_{K}^{m^{\prime}} N_{M_{0} / K} M_{0}^{\times}, \phi_{M / M_{0}}^{\left(\varphi^{d^{\prime}}\right)}\left(\left.\varphi^{-m} \sigma\right|_{M}\right)\right) \\
=\left(\pi_{K}^{m^{\prime}} N_{M_{0} / K} M_{0}^{\times}, \phi_{M / M_{0}}^{\left(\varphi^{d^{\prime}}\right)}\left(\varphi^{-m^{\prime}}(\sigma \mid M)\right)\right)=\phi_{M / K}^{(\varphi)}\left(\operatorname{res}_{M}(\sigma)\right)
\end{aligned}
$$

by Remark 2.2, part (i), which completes the proof.

Now, let $F / K$ be a finite subextension of $L / K$. Thus, $L / F$ is an infinite APF Galois extension (see Lemma 3.3 in [8]). We fix a Lubin-Tate splitting $\varphi_{F}$ over $F$ and assume that the residue-class degree $\left[\kappa_{L}: \kappa_{F}\right]$ is equal to $d^{\prime}$ for some $d^{\prime} \mid d$ and that there exists a chain of field extensions

$$
F \subset L \subset F_{\left(\varphi_{F}\right)^{d^{\prime}}}
$$


Thus, we have the generalized arrow

$$
\phi_{L / F}^{\left(\varphi_{F}\right)}: \operatorname{Gal}(L / F) \rightarrow F^{\times} / N_{L_{0}^{(F)} / F} L_{0}^{(F)^{\times}} \times U_{\widetilde{\mathbb{X}}(L / F)}^{\diamond} / U_{\mathbb{X}(L / F)}
$$

corresponding to the extension $L / F$. Here, as usual, $L_{0}^{(F)}$ is defined by $L_{0}^{(F)}=L \cap F^{n r}=$ $F_{d^{\prime}}^{n r}$. Observe the following diagram of field extensions:

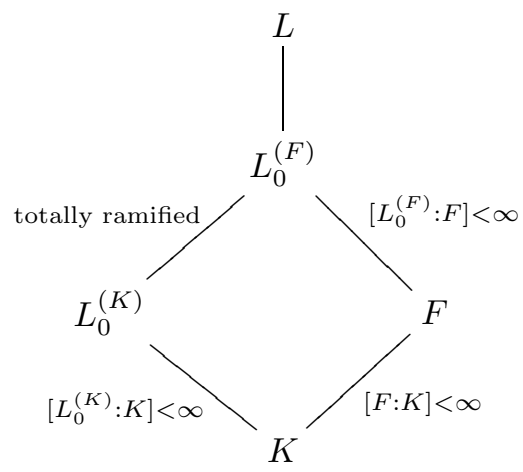

Thus, $L / L_{0}^{(F)}$ and $L / L_{0}^{(K)}$ are infinite totally ramified APF Galois extensions, by Lemma 3.3 in [8], and we have $L_{0}^{(F)} \subset L \subset\left(L_{0}^{(F)}\right)_{\varphi_{F}^{d^{\prime}}}$ and $L_{0}^{(K)} \subset L \subset\left(L_{0}^{(K)}\right)_{\varphi_{K}^{d}}$.

Remark 2.12. Note that $L_{0}^{(F)}$ is compatible with $\left(L_{0}^{(K)}, \varphi_{L_{0}^{(K)}}\right)$ in the sense of [10, p. 89], where $\varphi_{L_{0}^{(K)}}=\varphi_{K}^{d}$. Thus, $\varphi_{L_{0}^{(F)}}=\varphi_{F}^{d^{\prime}}=\varphi_{L_{0}^{(K)}}^{f\left(L_{0}^{(F)} / L_{0}^{(K)}\right)}=\varphi_{K}^{d}$, because $L_{0}^{(F)} / L_{0}^{(K)}$ is totally ramified.

For the extension $L / L_{0}^{(F)}$, we fix an ascending chain

$$
L_{0}^{(F)}=F_{o} \subset F_{1} \subset \cdots \subset F_{i} \subset \cdots \subset L
$$

satisfying $L=\bigcup_{0 \leq i \in \mathbb{Z}} F_{i}$ and $\left[F_{i+1}: F_{i}\right]<\infty$ for every $0 \leq i \in \mathbb{Z}$. Following [8] we introduce a homomorphism

$$
\Lambda_{F / K}: \widetilde{\mathbb{X}}\left(L / L_{0}^{(F)}\right)^{\times} \rightarrow \widetilde{\mathbb{X}}\left(L / L_{0}^{(K)}\right)^{\times}
$$

by

$$
\begin{aligned}
\Lambda_{F / K}:\left(\alpha_{F_{0}} \stackrel{\widetilde{N}_{F_{1} / F_{0}}}{\longleftarrow} \alpha_{F_{1}} \stackrel{\widetilde{N}_{F_{2} / F_{1}}}{\longleftarrow} \cdots\right) \\
\\
\mapsto\left(\widetilde{N}_{L_{0}^{(F)} / L_{0}^{(K)}}\left(\alpha_{F_{0}}\right) \stackrel{\widetilde{N}_{L_{0}^{(F)} / L_{0}^{(K)}}}{\longleftarrow} \alpha_{F_{0}} \stackrel{\widetilde{N}_{F_{1} / F}}{\longleftarrow} \alpha_{F_{1}} \stackrel{\widetilde{N}_{F_{2} / F_{1}}}{\longleftarrow} \cdots\right)
\end{aligned}
$$

for each $\left(\alpha_{F_{i}}\right)_{0 \leq i \in \mathbb{Z}} \in \widetilde{\mathbb{X}}\left(L / L_{0}^{(F)}\right)^{\times}$. This homomorphism induces a group homomorphism

$$
\lambda_{F / K}: U_{\widetilde{\mathbb{X}}\left(L / L_{0}^{(F)}\right)}^{\diamond} / U_{\mathbb{X}\left(L / L_{0}^{(F)}\right)} \rightarrow U_{\widetilde{\mathbb{X}}\left(L / L_{0}^{(K)}\right)}^{\diamond} / U_{\mathbb{X}\left(L / L_{0}^{(K)}\right)}
$$

defined by

$$
\lambda_{F / K}: \bar{U} \mapsto \Lambda_{F / K}(U) \cdot U_{\mathbb{X}\left(L / L_{0}^{(K)}\right)}
$$

for every $U \in U_{\widetilde{\mathbb{X}}\left(L / L_{0}^{(F)}\right)}^{\diamond}$, where the symbol $\bar{U}$ denotes the coset $U \cdot U_{\mathbb{X}\left(L / L_{0}^{(F)}\right)}$ in the group $U_{\widetilde{\mathbb{X}}\left(L / L_{0}^{(F)}\right)}^{\diamond} / U_{\mathbb{X}\left(L / L_{0}^{(F)}\right)}$ (see [8] for the details). 
Lemma 2.13. Let $F / K$ be a finite subextension of $L / K$. Fix a Lubin-Tate splitting $\varphi_{F}$ over $F$. Assume that the residue-class degree $\left[\kappa_{L}: \kappa_{F}\right]$ is equal to $d^{\prime}$ and $F \subset L \subset F_{\left(\varphi_{F}\right)^{d^{\prime}}}$ for some $d^{\prime} \mid d$. Then the square

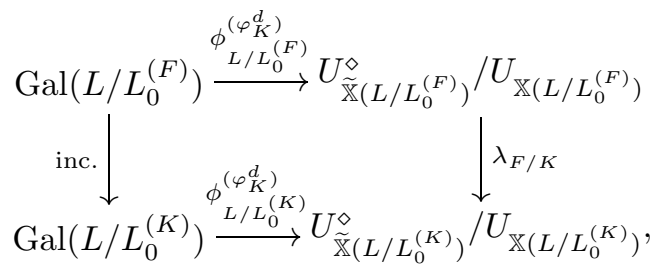

where the right vertical arrow

$$
\lambda_{F / K}: U_{\widetilde{\mathbb{X}}\left(L / L_{0}^{(F)}\right)}^{\diamond} / U_{\mathbb{X}\left(L / L_{0}^{(F)}\right)} \rightarrow U_{\widetilde{\mathbb{X}}\left(L / L_{0}^{(K)}\right)}^{\diamond} / U_{\mathbb{X}\left(L / L_{0}^{(K)}\right)}
$$

is defined by (2.27) and (2.28), is commutative.

Proof. Look at the proof of Theorem 5.12 in 8 .

We arrive at the following theorem.

Theorem 2.14. Let $F / K$ be a finite subextension of $L / K$. Fix a Lubin-Tate splitting $\varphi_{F}$ over $F$. Assume that the residue-class degree $\left[\kappa_{L}: \kappa_{F}\right]$ is equal to $d^{\prime}$ and $F \subset L \subset F_{\left(\varphi_{F}\right)^{d^{\prime}}}$ for some $d^{\prime} \mid d$. Then the following square is commutative:

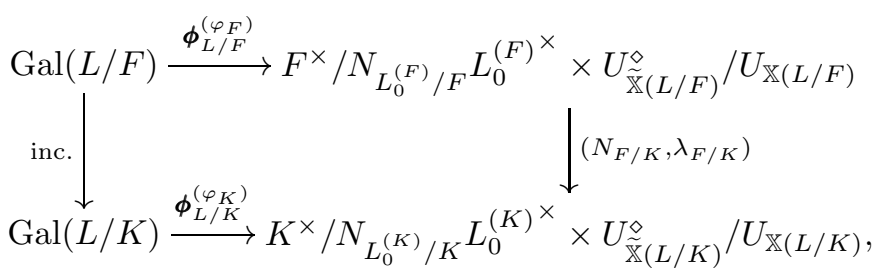

where the right vertical arrow

$$
\begin{aligned}
\left(N_{F / K}, \lambda_{F / K}\right): F^{\times} / N_{L_{0}^{(F)} / F} L_{0}^{(F)^{\times}} \times U_{\widetilde{\mathbb{X}}(L / F)}^{\diamond} / U_{\mathbb{X}(L / F)} & \\
& \rightarrow K^{\times} / N_{L_{0}^{(K)} / K} L_{0}^{(K)^{\times}} \times U_{\widetilde{\mathbb{X}}(L / K)} / U_{\mathbb{X}(L / K)}
\end{aligned}
$$

is defined by

$$
\left(N_{F / K}, \lambda_{F / K}\right):(\bar{a}, \bar{U}) \mapsto\left(\overline{N_{F / K}(a)}, \lambda_{F / K}(\bar{U})\right)
$$

for every $(\bar{a}, \bar{U}) \in F^{\times} / N_{L_{0}^{(F)} / F} L_{0}^{(F)^{\times}} \times U_{\widetilde{\mathbb{X}}(L / F)}^{\diamond} / U_{\mathbb{X}(L / F)}$.

Proof. Let $\sigma \in \operatorname{Gal}(L / F)$. There exists $0 \leq m \in \mathbb{Z}$ such that $\left.\sigma\right|_{L_{0}^{(F)}}=\varphi_{F}^{m}$ and $\varphi_{F}^{-m} \sigma \in$ $\operatorname{Gal}\left(L / L_{0}^{(F)}\right)$. Now,

$$
\boldsymbol{\phi}_{L / F}^{\left(\varphi_{F}\right)}(\sigma)=\left(\pi_{F}^{m} \cdot N_{L_{0}^{(F)} / F} L_{0}^{(F)^{\times}}, \phi_{L / L_{0}^{(F)}}^{\left(\varphi_{K}^{d}\right)}\left(\varphi_{F}^{-m} \sigma\right)\right)
$$

and

$$
\left(N_{F / K}, \lambda_{F / K}\right)\left(\phi_{L / F}^{\left(\varphi_{F}\right)}(\sigma)\right)=\left(\pi_{K}^{m} \cdot N_{L_{0}^{(K)} / K} L_{0}^{(K)^{\times}}, \phi_{L / L_{0}^{(K)}}^{\left(\varphi_{K}^{d}\right)}\left(\varphi_{F}^{-m} \sigma\right)\right)
$$

by the norm-compatibility of primes in the fixed Lubin-Tate labeling and by Lemma 2.13. There exists $0 \leq m^{\prime} \in \mathbb{Z}$ such that $\left.\sigma\right|_{L_{0}^{(K)}}=\varphi_{K}^{m^{\prime}}$ and $\varphi_{K}^{-m^{\prime}} \sigma \in \operatorname{Gal}\left(L / L_{0}^{(K)}\right)$. By part (ii) of Remark 2.2, it follows that $\left.\varphi_{F}^{m}\right|_{L_{0}^{(K)}}=\varphi_{K}^{m^{\prime}}$ and $\varphi_{F}^{-m} \sigma=\varphi_{K}^{-m^{\prime}} \sigma$. Now, 
$N_{F / K}: \pi_{F}^{m} N_{L_{0}^{(F)} / F} L_{0}^{(F)^{\times}} \mapsto \pi_{K}^{m^{\prime}} N_{L_{0}^{(K)} / K} L_{0}^{(K)^{\times}}=\pi_{K}^{m} \cdot N_{L_{0}^{(K)} / K} L_{0}^{(K)^{\times}}$by the Abelian local class field theory. Thus,

$$
\begin{aligned}
\left(N_{F / K}, \lambda_{F / K}\right)\left(\phi_{L / F}^{\left(\varphi_{F}\right)}(\sigma)\right) & =\left(\pi_{K}^{m} \cdot N_{L_{0}^{(K)} / K} L_{0}^{(K)^{\times}}, \phi_{L / L_{0}^{(K)}}^{\left(\varphi_{K}^{d}\right)}\left(\varphi_{F}^{-m} \sigma\right)\right) \\
& =\left(\pi_{K}^{m^{\prime}} \cdot N_{L_{0}^{(K)} / K} L_{0}^{(K)^{\times}}, \phi_{L / L_{0}^{(K)}}^{\left(\varphi_{K}^{d}\right)}\left(\varphi_{K}^{-m^{\prime}} \sigma\right)\right)=\phi_{L / K}^{\left(\varphi_{K}\right)}(\sigma),
\end{aligned}
$$

which completes the proof.

Let $L / K$ be any APF Galois subextension of $K_{\varphi^{d}} / K$, where the residue-class degree is $d$. If $L / K$ is assumed to be a finite extension, then the $\widetilde{K}$-coordinate of the generalized arrow $\phi_{L / K}^{(\varphi)}: \operatorname{Gal}(L / K) \rightarrow K^{\times} / N_{L_{0} / K} L_{0}^{\times} \times U_{\widetilde{\mathbb{X}}(L / K)}^{\diamond} / U_{\mathbb{X}(L / K)}$ is the Iwasawa-Neukirch map $\iota_{L / K}$ of $L / K$ (for the details on the Iwasawa-Neukirch map $\iota_{L / K}$ of the Galois extension $L / K$, see [8, §1]). More precisely, we have the following statement.

Proposition 2.15. Define a homomorphism

$$
\rho: K^{\times} / N_{L_{0} / K} L_{0}^{\times} \times U_{\widetilde{\mathbb{X}}(L / K)}^{\diamond} / U_{\mathbb{X}(L / K)} \rightarrow K^{\times} / N_{L / K} L^{\times}
$$

by

$$
\rho:\left(\pi_{K}^{m},\left(u_{\widetilde{E}}\right)\right) \mapsto \pi_{K}^{m} N_{L_{0} / K}\left(u_{\widetilde{L}_{0}}\right) \bmod N_{L / K} L^{\times}
$$

for every $\left(\pi_{K}^{m},\left(u_{\widetilde{E}}\right)\right) \in K^{\times} / N_{L_{0} / K} L_{0}^{\times} \times U_{\widetilde{\mathbb{X}}(L / K)}^{\diamond} / U_{\mathbb{X}(L / K)}$. Then the composite map

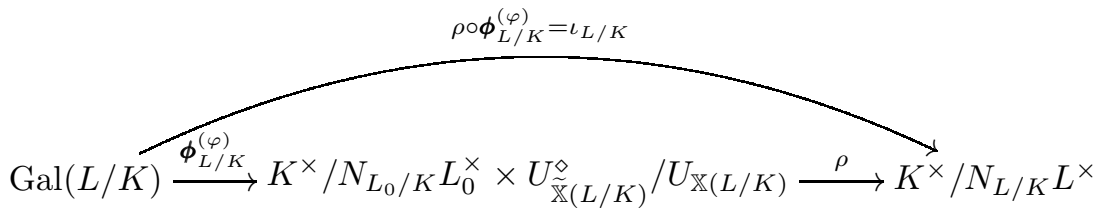

is the Iwasawa-Neukirch map $\iota_{L / K}: \operatorname{Gal}(L / K) \rightarrow K^{\times} / N_{L / K} L^{\times}$of $L / K$.

Proof. We follow $\S 1$ in 8$]$ to briefly recall the construction of the Iwasawa-Neukirch map

$$
\iota_{L / K}: \operatorname{Gal}(L / K) \rightarrow K^{\times} / N_{L / K} L^{\times}
$$

for the Galois extension $L / K$. For every $\sigma \in \operatorname{Gal}(L / K)$, we choose $\sigma^{*} \in \operatorname{Gal}\left(L^{n r} / K\right)$ in such a way that

(i) $\left.\sigma^{*}\right|_{L}=\sigma$, and

(ii) $\left.\sigma^{*}\right|_{K^{n r}=\varphi^{n}}$ for some $0<n \in \mathbb{Z}$.

Let $\Sigma_{\sigma^{*}}$ be the fixed field $\left(L^{n r}\right)^{\sigma^{*}}$ of $\sigma^{*} \in \operatorname{Gal}\left(L^{n r} / K\right)$ in $L^{n r}$. It is well known that $\left[\Sigma_{\sigma^{*}}: K\right]<\infty$. Now, the map $\iota_{L / K}: \operatorname{Gal}(L / K) \rightarrow K^{\times} / N_{L / K} L^{\times}$is defined by $\iota_{L / K}(\sigma)=$ $N_{\Sigma_{\sigma^{*}} / K}\left(\pi_{\Sigma_{\sigma^{*}}}\right) \bmod N_{L / K} L^{\times}$, for $\sigma \in \operatorname{Gal}(L / K)$, where $\pi_{\Sigma_{\sigma^{*}}}$ denotes any prime element of $\Sigma_{\sigma^{*}}$. Thus, for a finite APF Galois extension $L / K$ satisfying $\left[\kappa_{L}: \kappa_{K}\right]=d$ and $K \subset L \subset K_{\varphi^{d}}$, it suffices to prove that, for $\sigma \in \operatorname{Gal}(L / K)$,

$$
\rho \circ \phi_{L / K}^{(\varphi)}(\sigma)=\iota_{L / K}(\sigma)=N_{\Sigma_{\sigma^{*} / K}}\left(\pi_{\Sigma_{\sigma^{*}}}\right) \quad \bmod N_{L / K} L^{\times},
$$

where $\pi_{\Sigma_{\sigma^{*}}}$ denotes any prime element of $\Sigma_{\sigma^{*}}$. For $\sigma \in \operatorname{Gal}(L / K)$, there exists $0 \leq m \in \mathbb{Z}$ such that $\left.\sigma\right|_{L_{0}}=\varphi^{m}$ and $\tau=\varphi^{-m} \sigma \in \operatorname{Gal}\left(L / L_{0}\right)$. Thus, $\sigma=\varphi^{m} \tau$.

Case 1: $m>0$. In this case, it suffices to prove that

$$
\pi_{K}^{m} N_{L_{0} / K}\left(\operatorname{Pr}_{\widetilde{L}_{0}}\left(\phi_{L / L_{0}}^{\left(\varphi^{d}\right)}\left(\varphi^{-m} \sigma\right)\right)\right)=N_{\Sigma_{\sigma^{*}} / K}\left(\pi_{\Sigma_{\sigma^{*}}}\right) \quad \bmod N_{L / K} L^{\times}
$$


where $\pi_{\Sigma_{\sigma^{*}}}$ denotes any prime element of $\Sigma_{\sigma^{*}}$. To prove this relation, we choose $\sigma^{*} \in$ $\operatorname{Gal}\left(L^{n r} / K\right)$ such that

(i) $\left.\sigma^{*}\right|_{L}=\sigma$;

(ii) $\left.\sigma^{*}\right|_{K^{n r}}=\varphi^{m}$.

Indeed, let $\sigma^{*}=\left.\varphi^{m}\right|_{L^{n r}} \tau^{*}$, where $\tau^{*} \in \operatorname{Gal}\left(L^{n r} / L_{0}\right)$ is defined uniquely by the conditions $\left.\tau^{*}\right|_{L}=\tau$ and $\left.\tau^{*}\right|_{K^{n r}}=\mathrm{id}_{K^{n r}}$, because $L^{n r}=L K^{n r}$. Note that, for $\Sigma=\Sigma_{\sigma^{*}}$, $\Sigma_{0}=\Sigma \cap K^{n r}$ is a finite extension of degree $\left[\Sigma_{0}: K\right]=m$ over $K$, because $\Sigma_{0}$ coincides with $\left(K^{n r}\right)^{\varphi^{m}}$, the fixed field of $\varphi^{m} \in \operatorname{Gal}\left(K^{n r} / K\right)$ in $K^{n r}$. Since $L$ is fixed by $\varphi^{d}$, $T=L \cap K_{\varphi}$ is an unramified extension of degree $d$. Thus, the prime element $\pi_{T}$ is a prime element of $L$ and of $L^{n r}$. Now, choose a prime element $\pi_{\Sigma}$ of $\Sigma$. It is well known that $\Sigma^{n r}=L^{n r}$ (see $\S 2$ in Chapter 4 of 4 ). Thus, $\pi_{\Sigma}$ is a prime element of $L^{n r}$. So, there exists a unit $v \in L^{n r} \subset \widetilde{L}$ such that $\pi_{\Sigma}=\pi_{T} v$. Note that $\pi_{\Sigma}^{\sigma^{*}-1}=1$, because $\Sigma$ is fixed by $\sigma^{*}$. Thus, $\left(\pi_{T} v\right)^{\sigma^{*}-1}=1$ and we get the relations

$$
\pi_{T}^{\sigma-1}=v^{1-\sigma^{*}}=v^{1-\varphi^{m} \tau^{*}}=v^{1-\tau^{*}} v^{\left(1-\varphi^{m}\right) \tau^{*}} .
$$

Recall that (by Proposition 1.8 in Chapter IV of [4] or by Subsection 1.1 in [10]), $U_{\widetilde{L}}$ is multiplicatively $\left(1-\varphi^{m}\right)$-divisible. So, there exists $w \in U_{\widetilde{L}}$ such that $w^{1-\varphi^{m}}=v$. Hence,

$$
\pi_{T}^{\sigma-1}=\left(w^{1-\tau^{*}} v^{\tau^{*}}\right)^{1-\varphi^{m}},
$$

because $\varphi^{m} \tau^{*}=\tau^{*} \varphi^{m}$. Now, we choose $z \in U_{\widetilde{L}}$ as follows: $z=\left(w^{1-\tau^{*}} v^{\tau^{*}}\right)^{1+\varphi+\cdots+\varphi^{m-1}}$. Observe that this $z \in U_{\widetilde{L}}$ satisfies $z^{1-\varphi}=\pi_{T}^{\sigma-1}$. Clearly,

$$
\widetilde{N}_{L / K}(z)=\widetilde{N}_{L / K}(v)^{1+\varphi+\cdots+\varphi^{m-1}} .
$$

After these preliminary observations, we see that

$$
\begin{aligned}
N_{\Sigma / K}\left(\pi_{\Sigma}\right) & =N_{\Sigma_{0} / K} \circ N_{\Sigma / \Sigma_{0}}\left(\pi_{\Sigma}\right) \\
& =\widetilde{N}_{L / K}\left(\pi_{\Sigma}\right)^{1+\varphi+\cdots+\varphi^{m-1}} \\
& =\widetilde{N}_{L / K}\left(\pi_{T} v\right)^{1+\sigma+\cdots+\sigma^{m-1}} \\
& =\pi_{K}^{m} \widetilde{N}_{L / K}(v)^{1+\varphi+\cdots+\varphi^{m-1}} \\
& =\pi_{K}^{m} \widetilde{N}_{L / K}(z),
\end{aligned}
$$

because $\pi_{T}$ belongs to the fixed Lubin-Tate labeling. Thus, the image of $\sigma$ under the Iwasawa-Neukirch map $\iota_{L / K}$ is

$$
\iota_{L / K}(\sigma)=\pi_{K}^{m} \tilde{N}_{L / K}(z) \bmod N_{L / K} L^{\times} .
$$

Now, let $y \in U_{L}$ be such that

$$
y^{1-\varphi^{d}}=\pi_{T}^{\varphi^{-m} \sigma-1}=\pi_{T}^{\sigma-1} .
$$

Note that $T=L \cap K_{\varphi}$. Then, setting $z=y^{1+\varphi+\cdots+\varphi^{d-1}} \in U_{L}$, we have

$$
z^{1-\varphi}=y^{1-\varphi^{d}}=\pi_{T}^{\sigma-1} .
$$

Thus,

$$
N_{L / K}(y)=\widetilde{N}_{L / K}(y)^{1+\varphi+\cdots+\varphi^{d-1}}=\widetilde{N}_{L / K}(z),
$$


which shows that

$$
\begin{aligned}
\iota_{L / K}(\sigma) & =\pi_{K}^{m} \widetilde{N}_{L / K}(z) \quad \bmod N_{L / K} L^{\times} \\
& =\pi_{K}^{m} N_{L / K}(y) \quad \bmod N_{L / K} L^{\times} \\
& =\pi_{K}^{m} N_{L_{0} / K}\left(\widetilde{N}_{L / K}(y)\right) \quad \bmod N_{L / K} L^{\times} \\
& =\pi_{K}^{m} N_{L_{0} / K}\left(\operatorname{Pr}_{\widetilde{L}_{0}}\left(\phi_{L / L_{0}}^{\left(\varphi^{d}\right)}\left(\varphi^{-m} \sigma\right)\right)\right) \\
& =\rho \circ \phi_{L / K}^{(\varphi)}(\sigma),
\end{aligned}
$$

completing the proof.

Case 2: $m=0$. In this case, $\sigma \in \operatorname{Gal}\left(L / L_{0}\right)$. Consider $\varphi^{d} \sigma \in \operatorname{Gal}\left(L^{n r} / K\right)$. Then, by Case 1, we have

$$
\iota_{L / K}\left(\varphi^{d} \sigma\right)=\rho \circ \phi_{L / K}^{(\varphi)}\left(\varphi^{d} \sigma\right),
$$

where $\iota_{L / K}\left(\varphi^{d} \sigma\right)=\iota_{L / K}(\sigma)$. Theorem 2.4 implies the formulas

$$
\boldsymbol{\phi}_{L / K}^{(\varphi)}\left(\varphi^{d} \sigma\right)=\boldsymbol{\phi}_{L / K}^{(\varphi)}\left(\varphi^{d}\right) \boldsymbol{\phi}_{L / K}^{(\varphi)}(\sigma)^{\varphi^{d}}=\left(\pi_{K}^{d} \cdot N_{L_{0} / K} L_{0}^{\times}, \phi_{L / L_{0}}^{\left(\varphi^{d}\right)}(\sigma)\right),
$$

where the last identity follows from the fact that $K \subset L \subset K_{\varphi^{d}}$. Thus,

$$
\rho \circ \phi_{L / K}^{(\varphi)}(\sigma)=\rho \circ \boldsymbol{\phi}_{L / K}^{(\varphi)}\left(\varphi^{d} \sigma\right)=\pi_{K}^{d} N_{L_{0} / K}\left(\operatorname{Pr}_{\widetilde{L}_{0}}\left(\phi_{L / L_{0}}^{\left(\varphi^{d}\right)}(\sigma)\right)\right) \quad \bmod N_{L / K} L^{\times},
$$

which proves the relation

$$
\iota_{L / K}(\sigma)=\rho \circ \boldsymbol{\phi}_{L / K}^{(\varphi)}(\sigma) .
$$

The proposition is proved.

Now, we generalize Fesenko's definition of the extended Hazewinkel map $H_{L / K}^{(\varphi)}$ : $U_{\widetilde{\mathbb{X}}(L / K)}^{\diamond} / Y_{L / K} \rightarrow \operatorname{Gal}(L / K)$ (cf. [1, 2, 3] and [8]), initially defined for totally ramified APF Galois subextensions $L / K$ of $K_{\varphi} / K$, to infinite APF Galois subextensions $L / K$ of $K_{\varphi^{d}} / K$, where $\left[\kappa_{L}: \kappa_{K}\right]=d$.

For this, first we assume that the local field $K$ satisfies the condition

$$
\boldsymbol{\mu}_{p}\left(K^{\mathrm{sep}}\right)=\left\{\alpha \in K^{\mathrm{sep}}: \alpha^{p}=1\right\} \subset K,
$$

where $p=\operatorname{char}\left(\kappa_{K}\right)$. For the details on the assumption (2.34) on $K$, we refer the reader to $[1,2,[3$.

Let $L / K$ be an infinite APF Galois extension with residue-class degree $\left[\kappa_{L}: \kappa_{K}\right]=d$ and with $K \subset L \subset K_{\varphi^{d}}$. As usual, let $L_{0}=L \cap K^{n r}$. We introduce the generalized arrow

$$
\boldsymbol{H}_{L / K}^{(\varphi)}: K^{\times} / N_{L_{0} / K} L_{0}^{\times} \times U_{\widetilde{\mathbb{X}}(L / K)}^{\diamond} / Y_{L / L_{0}} \rightarrow \operatorname{Gal}(L / K)
$$

for the extension $L / K$ by

$$
\boldsymbol{H}_{L / K}^{(\varphi)}\left(\left(\pi_{K}^{m} N_{L_{0} / K} L_{0}^{\times}, U . Y_{L / L_{0}}\right)\right)=\left.\varphi^{m}\right|_{L} H_{L / L_{0}}^{\left(\varphi^{d}\right)}\left(U . Y_{L / L_{0}}\right)
$$

for every $m \in \mathbb{Z}$ and every $U \in U_{\widetilde{\mathbb{X}}(L / K)}^{\diamond}$, where $H_{L / L_{0}}^{\left(\varphi^{d}\right)}: U_{\widetilde{\mathbb{X}}(L / K)}^{\diamond} / Y_{L / L_{0}} \rightarrow \operatorname{Gal}\left(L / L_{0}\right)$ is the extended Hazewinkel map of Fesenko for the extension $L / L_{0}$. For the definition and basic properties of the group $Y_{L / L_{0}}$, we refer the reader to [3] and [8].

The following lemma is clear. 
Lemma 2.16. Suppose that the local field $K$ satisfies condition (2.34). Let $L / K$ be an infinite APF Galois subextension of $K_{\varphi^{d}} / K$, where $d=\left[\kappa_{L}: \kappa_{K}\right]$. Then the generalized arrow

$$
\boldsymbol{H}_{L / K}^{(\varphi)}: K^{\times} / N_{L_{0} / K} L_{0}^{\times} \times U_{\widetilde{\mathbb{X}}(L / K)}^{\diamond} / Y_{L / L_{0}} \rightarrow \operatorname{Gal}(L / K)
$$

for the extension $L / K$ is a bijection.

Proof. The proof follows from the isomorphism

$$
\operatorname{Gal}(L / K) \simeq \operatorname{Gal}\left(L_{0} / K\right) \times \operatorname{Gal}\left(L / L_{0}\right)
$$

combined with the bijectivity of

$$
H_{L / L_{0}}^{\left(\varphi^{d}\right)}: U_{\widetilde{\mathbb{X}}(L / K)}^{\diamond} / Y_{L / L_{0}} \rightarrow \operatorname{Gal}\left(L / L_{0}\right)
$$

(cf. Lemma 5.22 of [8]) and the Abelian local class field theory for the extension $L_{0} / K$.

Now, consider the composition of arrows

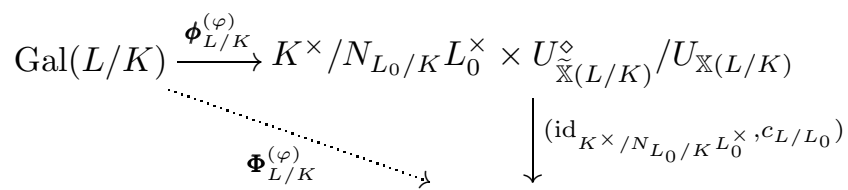

$$
\begin{aligned}
& K^{\times} / N_{L_{0} / K} L_{0}^{\times} \times U_{\widetilde{\mathbb{X}}(L / K)}^{\diamond} / Y_{L / L_{0}},
\end{aligned}
$$

where $c_{L / L_{0}}: U_{\widetilde{\mathbb{X}}(L / K)}^{\diamond} / U_{\mathbb{X}(L / K)} \rightarrow U_{\widetilde{\mathbb{X}}(L / K)}^{\diamond} / Y_{L / L_{0}}$ is the canonical map defined via the inclusion $U_{\mathbb{X}(L / K)} \subseteq Y_{L / L_{0}}$. Recall that (see [8, (5.35)]) the composition $c_{L / L_{0}} \circ \phi_{L / L_{0}}^{\left(\varphi^{d}\right)}=$ $\Phi_{L / L_{0}}^{\left(\varphi^{d}\right)}: \operatorname{Gal}\left(L / L_{0}\right) \rightarrow U_{\widetilde{\mathbb{X}}(L / K)}^{\diamond} / Y_{L / L_{0}}$ is the Fesenko reciprocity map for the extension $L / L_{0}$. Now, let $\sigma \in \operatorname{Gal}(L / K)$. Let $0 \leq m \in \mathbb{Z}$ be such that $\left.\sigma\right|_{L_{0}}=\left.\varphi^{m}\right|_{L_{0}}$ and $\varphi^{-m} \sigma \in \operatorname{Gal}\left(L / L_{0}\right)$. Then, in accordance with the definition,

$$
\begin{aligned}
\boldsymbol{H}_{L / K}^{(\varphi)} \circ \boldsymbol{\Phi}_{L / K}^{(\varphi)}(\sigma) & =\boldsymbol{H}_{L / K}^{(\varphi)}\left(\pi_{K}^{m} \cdot N_{L_{0} / K} L_{0}^{\times}, c_{L / L_{0}} \circ \phi_{L / L_{0}}^{\left(\varphi^{d}\right)}\left(\varphi^{-m} \sigma\right)\right) \\
& =\boldsymbol{H}_{L / K}^{(\varphi)}\left(\pi_{K}^{m} \cdot N_{L_{0} / K} L_{0}^{\times}, \Phi_{L / L_{0}}^{\left(\varphi^{d}\right)}\left(\varphi^{-m} \sigma\right)\right) \\
& =\left.\varphi^{m}\right|_{L} H_{L / L_{0}}^{\left(\varphi^{d}\right)}\left(\Phi_{L / L_{0}}^{\left(\varphi^{d}\right)}\left(\varphi^{-m} \sigma\right)\right) \\
& =\left.\varphi^{m}\right|_{L}\left(\varphi^{-m} \sigma\right) \\
& =\sigma
\end{aligned}
$$

by [8, Lemma 5.23]. For $0 \leq m \in \mathbb{Z}$ and $U \in U_{\widetilde{\mathbb{X}}(L / K)}^{\diamond}$, let

$$
\left(\pi_{K}^{m} \cdot N_{L_{0} / K} L_{0}^{\times}, U \cdot Y_{L / L_{0}}\right) \in K^{\times} / N_{L_{0} / K} L_{0}^{\times} \times U_{\widetilde{\mathbb{X}}(L / K)}^{\diamond} / Y_{L / L_{0}} .
$$

Now, again by definition,

$$
\begin{aligned}
\boldsymbol{\Phi}_{L / K}^{(\varphi)} \circ \boldsymbol{H}_{L / K}^{(\varphi)}\left(\left(\pi_{K}^{m} \cdot N_{L_{0} / K} L_{0}^{\times}, U \cdot Y_{L / L_{0}}\right)\right)=\boldsymbol{\Phi}_{L / K}^{(\varphi)}\left(\left.\varphi^{m}\right|_{L} H_{L / L_{0}}^{\left(\varphi^{d}\right)}\left(U \cdot Y_{L / L_{0}}\right)\right) \\
=\left(\operatorname{id}_{K^{\times} / N_{L_{0} / K} L_{0}^{\times}}, c_{L / L_{0}}\right) \circ \phi_{L / K}^{(\varphi)}\left(\left.\varphi^{m}\right|_{L} H_{L / L_{0}}^{\left(\varphi^{d}\right)}\left(U \cdot Y_{L / L_{0}}\right)\right) \\
=\left(\operatorname{id}_{K^{\times} / N_{L_{0} / K} L_{0}^{\times}}, c_{L / L_{0}}\right)\left(\pi_{K}^{m} \cdot N_{L_{0} / K} L_{0}^{\times}, \phi_{L / L_{0}}^{\left(\varphi^{d}\right)}\left(H_{L / L_{0}}^{\left(\varphi^{d}\right)}\left(U \cdot Y_{L / L_{0}}\right)\right)\right. \\
=\left(\pi_{K}^{m} \cdot N_{L_{0} / K} L_{0}^{\times}, \Phi_{L / L_{0}}^{\left(\varphi^{d}\right)}\left(H_{L / L_{0}}^{\left(\varphi^{d}\right)}\left(U \cdot Y_{L / L_{0}}\right)\right)\right. \\
=\left(\pi_{K}^{m} \cdot N_{L_{0} / K} L_{0}^{\times}, U \cdot Y_{L / L_{0}}\right),
\end{aligned}
$$


by [8, Lemma 5.23]. These computations yield

$$
\boldsymbol{H}_{L / K}^{(\varphi)} \circ \boldsymbol{\Phi}_{L / K}^{(\varphi)}=\operatorname{id}_{\mathrm{Gal}(L / K)}
$$

and

$$
\boldsymbol{\Phi}_{L / K}^{(\varphi)} \circ \boldsymbol{H}_{L / K}^{(\varphi)}=\operatorname{id}_{K \times / N_{L_{0} / K} L_{0}^{\times} \times U_{\tilde{\mathbb{X}}(L / K)}^{\diamond} / Y_{L / L_{0}}} .
$$

Note that there is a natural continuous action of $\operatorname{Gal}(L / K)$ on the topological group $K^{\times} / N_{L_{0} / K} L_{0}^{\times} \times U_{\widetilde{\mathbb{X}}(L / K)}^{\diamond} / Y_{L / L_{0}}$, defined by the Abelian local class field theory on the first component and by formula (5.7) and Lemma 5.20 in [8] on the second component:

$$
(\bar{a}, \bar{U})^{\sigma}=\left(\bar{a}^{\varphi^{m}}, \bar{U}^{\varphi^{-m} \sigma}\right)=\left(\bar{a}, \bar{U}^{\varphi^{-m} \sigma}\right),
$$

for every $\sigma \in \operatorname{Gal}(L / K)$, where $\left.\sigma\right|_{L_{0}}=\varphi^{m}$ for some $0 \leq m \in \mathbb{Z}$ and for every $a \in K^{\times}$ with $\bar{a}=a \cdot N_{L_{0} / K} L_{0}^{\times}$and $U \in U_{\widetilde{\mathbb{X}}(L / K)}^{\diamond}$ with $\bar{U}=U \cdot Y_{L / L_{0}}$. We shall always view $K^{\times} / N_{L_{0} / K} L_{0}^{\times} \times U_{\widetilde{\mathbb{X}}(L / K)}^{\diamond} / Y_{L / L_{0}}$ as a topological Gal $(L / K)$-module in this paper.

So, we arrive at the following theorem, which follows from Theorem 2.4. Lemma 2.16. and relations (2.39) and (2.40) combined with the fact that $U_{\mathbb{X}(L / K)}$ is a topological $\operatorname{Gal}\left(L / L_{0}\right)$-submodule of $Y_{L / L_{0}}$.

Theorem 2.17. Suppose that the local field $K$ satisfies condition (2.34). Let $L / K$ be an infinite APF Galois subextension of $K_{\varphi^{d}} / K$, where $d=\left[\kappa_{L}: \kappa_{K}\right]$. The mapping

$$
\boldsymbol{\Phi}_{L / K}^{(\varphi)}: \operatorname{Gal}(L / K) \rightarrow K^{\times} / N_{L_{0} / K} L_{0}^{\times} \times U_{\widetilde{\mathbb{X}}(L / K)}^{\diamond} / Y_{L / L_{0}}
$$

defined for the extension $L / K$ is a bijection with the inverse

$$
H_{L / K}^{(\varphi)}: K^{\times} / N_{L_{0} / K} L_{0}^{\times} \times U_{\widetilde{\mathbb{X}}(L / K)}^{\diamond} / Y_{L / L_{0}} \rightarrow \operatorname{Gal}(L / K) .
$$

For every $\sigma, \tau \in \operatorname{Gal}(L / K)$, the cocycle condition

$$
\boldsymbol{\Phi}_{L / K}^{(\varphi)}(\sigma \tau)=\boldsymbol{\Phi}_{L / K}^{(\varphi)}(\sigma) \boldsymbol{\Phi}_{L / K}^{(\varphi)}(\tau)^{\sigma}
$$

is satisfied.

By Corollary 2.5. Theorem 2.17 has the following consequence.

Corollary 2.18. Suppose that a law of composition $*$ is defined on the set $K^{\times} / N_{L_{0} / K} L_{0}^{\times}$ $\times U_{\widetilde{\mathbb{X}}(L / K)}^{\diamond} / Y_{L / L_{0}}$ by the rule

$$
(\bar{a}, \bar{U}) *(\bar{b}, \bar{V})=(\bar{a}, \bar{U}) \cdot(\bar{b}, \bar{V})^{\left(\Phi_{L / K}^{(\varphi)}\right)^{-1}((\bar{a}, \bar{U}))}
$$

for every $\bar{a}=a \cdot N_{L_{0} / K} L_{0}^{\times}, \bar{b}=b \cdot N_{L_{0} / K} L_{0}^{\times} \in K^{\times} / N_{L_{0} / K} L_{0}^{\times}$with $a, b \in K^{\times}$and every $\bar{U}=U . Y_{L / L_{0}}$ and $\bar{V}=V . Y_{L / L_{0}} \in U_{\widetilde{\mathbb{X}}(L / K)}^{\diamond} / Y_{L / L_{0}}$ such that $U, V \in U_{\widetilde{\mathbb{X}}(L / K)}^{\diamond}$. Then $K^{\times} / N_{L_{0} / K} L_{0}^{\times} \times U_{\widetilde{\mathbb{X}}(L / K)}^{\diamond} / Y_{L / L_{0}}$ is a topological group under $*$, and the map $\boldsymbol{\Phi}_{L / K}^{(\varphi)}$ induces an isomorphism of topological groups

$$
\boldsymbol{\Phi}_{L / K}^{(\varphi)}: \operatorname{Gal}(L / K) \stackrel{\sim}{\longrightarrow} K^{\times} / N_{L_{0} / K} L_{0}^{\times} \times U_{\widetilde{\mathbb{X}}(L / K)}^{\diamond} / Y_{L / L_{0}},
$$

where the topological group structure on $K^{\times} / N_{L_{0} / K} L_{0}^{\times} \times U_{\widetilde{\mathbb{X}}(L / K)}^{\diamond} / Y_{L / L_{0}}$ is defined with respect to the binary operation * introduced by (2.43).

Definition 2.19. Let $K$ be a local field satisfying condition (2.34). Let $L / K$ be an infinite APF Galois subextension of $K_{\varphi^{d}} / K$, where $d=\left[\kappa_{L}: \kappa_{K}\right]$. The mapping

$$
\boldsymbol{\Phi}_{L / K}^{(\varphi)}: \operatorname{Gal}(L / K) \rightarrow K^{\times} / N_{L_{0} / K} L_{0}^{\times} \times U_{\widetilde{\mathbb{X}}(L / K)}^{\diamond} / Y_{L / L_{0}}
$$


defined in Theorem 2.17 is called the generalized Fesenko reciprocity map for the extension $L / K$.

We recall that, for each $0 \leq i \in \mathbb{R}$, the higher unit groups $\left(U_{\widetilde{\mathbb{X}}(L / K)}^{\diamond}\right)^{i}$ of the field $\widetilde{\mathbb{X}}(L / K)$ were introduced in (2.16). As in [8, (5.42)], for each $0 \leq n \in \mathbb{Z}$ we put

$$
Q_{L / L_{0}}^{n}=c_{L / L_{0}}\left(\left(U_{\widetilde{\mathbb{X}}(L / K)}^{\diamond}\right)^{n} U_{\mathbb{X}(L / K)} / U_{\mathbb{X}(L / K)} \cap \operatorname{im}\left(\phi_{L / L_{0}}^{\left(\varphi^{d}\right)}\right)\right) ;
$$

this is a subgroup of $\left(U_{\widetilde{\mathbb{X}}(L / K)}^{\diamond}\right)^{n} Y_{L / L_{0}} / Y_{L / L_{0}}$. Now, the ramification statement of Theorem 2.7 can be reformulated for the generalized reciprocity map $\Phi_{L / K}^{(\varphi)}$ corresponding to the extension $L / K$ as follows.

Theorem 2.20 (Ramification theorem). Let $K$ be a local field satisfying condition (2.34). For $0 \leq u \in \mathbb{R}$, let $\operatorname{Gal}(L / K)_{u}$ denote the uth higher ramification subgroup in the lower numbering of the Galois group $\operatorname{Gal}(L / K)$ corresponding to the infinite APF Galois subextension $L / K$ of $K_{\varphi^{d}} / K$ with residue-class degree $\left[\kappa_{L}: \kappa_{K}\right]$ equal to d. Then, for $0 \leq n \in \mathbb{Z}$, we have

$$
\begin{aligned}
\boldsymbol{\Phi}_{L / K}^{(\varphi)}\left(\operatorname{Gal}(L / K)_{\psi_{L / K} \circ \varphi_{L / L_{0}}(n)}-\operatorname{Gal}(L / K)_{\psi_{L / K} \circ \varphi_{L / L_{0}}(n+1)}\right) \\
\subseteq\left\langle 1_{K^{\times} / N_{L_{0} / K} L_{0}^{\times}}\right\rangle \times\left(\left(U_{\widetilde{\mathbb{X}}(L / K)}^{\diamond}\right)^{n} Y_{L / L_{0}} / Y_{L / L_{0}}-Q_{L / L_{0}}^{n+1}\right) .
\end{aligned}
$$

Proof. In accordance with the general observation made in the first paragarph of the proof of Theorem 2.7, for $0 \leq u \in \mathbb{R}$ and for $\tau \in \operatorname{Gal}(L / K)_{u}=\operatorname{Gal}\left(L / L_{0}\right)^{\varphi_{L / K}(u)}$ we have

$$
\phi_{L / K}^{(\varphi)}(\tau)=\left(1_{K^{\times} / N_{L_{0} / K} L_{0}^{\times}}, \phi_{L / L_{0}}^{\left(\varphi^{\prime}\right)}(\tau)\right)
$$

where $\varphi^{\prime}=\varphi^{d}$. Thus, by the definition,

$$
\boldsymbol{\Phi}_{L / K}^{(\varphi)}(\tau)=\left(1_{K^{\times} / N_{L_{0} / K} L_{0}^{\times}}, c_{L / L_{0}} \circ \phi_{L / L_{0}}^{\left(\varphi^{\prime}\right)}(\tau)\right)=\left(1_{K^{\times} / N_{L_{0} / K} L_{0}^{\times}}, \Phi_{L / L_{0}}^{\left(\varphi^{\prime}\right)}(\tau)\right) .
$$

Now, to prove the theorem, we take $u=\psi_{L / K} \circ \varphi_{L / L_{0}}(n)$ and $u^{\prime}=\psi_{L / K} \circ \varphi_{L / L_{0}}(n+1)$. Then, the ramification theorem (see [8, Theorem 5.27]) shows that, for any $\tau \in \operatorname{Gal}(L / K)_{u}$ $-\operatorname{Gal}(L / K)_{u^{\prime}}$ the second coordinate of $\boldsymbol{\Phi}_{L / K}^{(\varphi)}(\tau)$ satisfies

$$
\Phi_{L / L_{0}}^{\left(\varphi^{\prime}\right)}(\tau) \in\left(U_{\widetilde{\mathbb{X}}(L / K)}^{\diamond}\right)^{n} Y_{L / L_{0}} / Y_{L / L_{0}}-Q_{L / L_{0}}^{n+1}
$$

because

$$
\operatorname{Gal}(L / K)_{u}=\operatorname{Gal}\left(L / L_{0}\right)^{\varphi_{L / K}(u)}=\operatorname{Gal}\left(L / L_{0}\right)^{\varphi_{L / L_{0}}(n)}=\operatorname{Gal}\left(L / L_{0}\right)_{n}
$$

and likewise

$$
\operatorname{Gal}(L / K)_{u^{\prime}}=\operatorname{Gal}\left(L / L_{0}\right)^{\varphi_{L / K}\left(u^{\prime}\right)}=\operatorname{Gal}\left(L / L_{0}\right)^{\varphi_{L / L_{0}}(n+1)}=\operatorname{Gal}\left(L / L_{0}\right)_{n+1},
$$

which completes the proof.

Let $K$ be a local field satisfying condition (2.34). Let $M / K$ be an infinite Galois subextension of $L / K$. Thus, by [8, Lemma 3.3], $M$ is an APF Galois extension over $K$. We further assume that the residue-class degree $\left[\kappa_{M}: \kappa_{K}\right]$ is equal to $d^{\prime}$ and $K \subset M \subset$ $K_{\varphi^{d^{\prime}}}$ for some $d^{\prime} \mid d$. Let

$$
\Phi_{M / K}^{(\varphi)}: \operatorname{Gal}(M / K) \rightarrow K^{\times} / N_{M_{0} / K} M_{0}^{\times} \times U_{\widetilde{\mathbb{X}}(M / K)}^{\diamond} / Y_{M / M_{0}}
$$


be the corresponding generalized Fesenko reciprocity map defined for the extension $M / K$. Here, as usual, $M_{0}$ is defined by $M_{0}=M \cap K^{n r}=K_{d^{\prime}}^{n r}$. Now, we fix a basic sequence

$$
L_{o}=E_{o} \subset E_{1} \subset \cdots \subset E_{i} \subset \cdots \subset L
$$

for the extension $L / L_{0}$. Using the notation of [3] and [8], for each $1 \leq i \in \mathbb{Z}$ we introduce an element $\sigma_{i}$ in $\operatorname{Gal}(\widetilde{L} / \widetilde{K})$ such that $\left\langle\left.\sigma\right|_{E_{i}}\right\rangle=\operatorname{Gal}\left(E_{i} / E_{i-1}\right)$. Next, for each $1 \leq k, i \in \mathbb{Z}$, we introduce the map

the map

$$
h_{k}^{\left(L / L_{o}\right)}: \prod_{1 \leq i \leq k} U_{\widetilde{E}_{k}}^{\sigma_{i}-1} \rightarrow\left(\prod_{1 \leq i \leq k+1} U_{\widetilde{E}_{k+1}}^{\sigma_{i}-1}\right) / U_{\widetilde{E}_{k+1}}^{\sigma_{k+1}-1},
$$

$$
g_{k}^{\left(L / L_{o}\right)}: \prod_{1 \leq i \leq k} U_{\widetilde{E}_{k}}^{\sigma_{i}-1} \rightarrow \prod_{1 \leq i \leq k+1} U_{\widetilde{E}_{k+1}}^{\sigma_{i}-1}
$$

and the map

$$
f_{i}^{\left(L / L_{o}\right)}: U_{\widetilde{E}_{i}}^{\sigma_{i}-1} \rightarrow U_{\widetilde{\mathbb{X}}\left(L / E_{i}\right)} \stackrel{\Lambda_{E_{i} / E_{o}}}{\longrightarrow} U_{\widetilde{\mathbb{X}}(L / K)}
$$

as in [3] and [8]. We fix the sequence

$$
M_{o}=E_{o} \cap M \subseteq E_{1} \cap M \subseteq \cdots \subseteq E_{i} \cap M \subseteq \cdots \subseteq M
$$

for the extension $M / M_{o}$ and, for each $1 \leq k \in \mathbb{Z}$, define a homomorphism

$$
h_{k}^{\left(M / M_{o}\right)}: \prod_{1 \leq i \leq k} U_{\overparen{E_{k} \cap M}}^{\left.\sigma_{i}\right|_{\widetilde{M}}-1} \rightarrow\left(\prod_{1 \leq i \leq k+1} U_{E_{k+1}^{\left.\sigma_{i} \cap\right|_{\overparen{M}}-1}}^{\overbrace{1} \cap M}\right) / U_{E_{k+1} \cap M}^{\left.\sigma_{k+1}\right|_{\widetilde{M}}-1}
$$

that satisfies

$$
\begin{aligned}
\left(\prod_{0 \leq \ell \leq f(L / M)-1}\left(\varphi^{d^{\prime}}\right)^{\ell} \widetilde{N}_{E_{k+1} / E_{k+1} \cap M}\right) \circ h_{k}^{\left(L / L_{o}\right)} & \\
& =h_{k}^{\left(M / M_{o}\right)} \circ\left(\prod_{0 \leq \ell \leq f(L / M)-1}\left(\varphi^{d^{\prime}}\right)^{\ell} \widetilde{N}_{E_{k} / E_{k} \cap M}\right)
\end{aligned}
$$

and take any map

that satisfies

$$
g_{k}^{\left(M / M_{o}\right)}: \prod_{1 \leq i \leq k} U_{E_{k} \cap M}^{\left.\sigma_{i}\right|_{\widetilde{M}}-1} \rightarrow \prod_{1 \leq i \leq k+1} U_{\left.E_{k+1} \cap\right|_{\overparen{\widetilde{M}}}-1}^{\sigma_{k+1} \cap}
$$

$$
\begin{aligned}
\left(\prod_{0 \leq \ell \leq f(L / M)-1}\left(\varphi^{d^{\prime}}\right)^{\ell} \widetilde{N}_{E_{k+1} / E_{k+1} \cap M}\right) \circ g_{k}^{\left(L / L_{o}\right)} & \\
& =g_{k}^{\left(M / M_{o}\right)} \circ\left(\prod_{0 \leq \ell \leq f(L / M)-1}\left(\varphi^{d^{\prime}}\right)^{\ell} \widetilde{N}_{E_{k} / E_{k} \cap M}\right),
\end{aligned}
$$

again following the same lines of $[3]$ and $[8$.

Now, for each $1 \leq i \in \mathbb{Z}$, we introduce a map

$$
f_{i}^{\left(M / M_{o}\right)}: U_{\overparen{E_{i} \cap M}}^{\left.\sigma_{i}\right|_{\widetilde{M}}-1} \rightarrow U_{\widetilde{\mathbb{X}}(M / K)}
$$

by

$$
f_{i}^{\left(M / M_{o}\right)}(w)=\widetilde{\mathcal{N}}_{L / M}\left(f_{i}^{\left(L / L_{o}\right)}(v)\right),
$$

where $v \in U_{\widetilde{E}_{i}}^{\sigma_{i}-1}$ is any element satisfying

$$
\prod_{0 \leq \ell \leq f(L / M)-1}\left(\varphi^{d^{\prime}}\right)^{\ell} \widetilde{N}_{E_{i} / E_{i} \cap M}(v)=w \in U_{\overparen{E_{i} \cap M}}^{\left.\sigma_{i}\right|_{\widetilde{M}}-1} .
$$


Observe that if $v^{\prime} \in U_{\widetilde{E}_{i}}^{\sigma_{i}-1}$ is such that

$$
\prod_{0 \leq \ell \leq f(L / M)-1}\left(\varphi^{d^{\prime}}\right)^{\ell} \widetilde{N}_{E_{i} / E_{i} \cap M}\left(v^{\prime}\right)=w
$$

then

$$
\widetilde{\mathcal{N}}_{L / M}\left(f_{i}^{\left(L / L_{o}\right)}(v)\right)=\widetilde{\mathcal{N}}_{L / M}\left(f_{i}^{\left(L / L_{o}\right)}\left(v^{\prime}\right)\right) .
$$

Indeed, there exists $u \in \operatorname{ker}\left(\prod_{0 \leq \ell \leq f(L / M)-1}\left(\varphi^{d^{\prime}}\right)^{\ell} \widetilde{N}_{E_{i} / E_{i} \cap M}\right)$ such that $v^{\prime}=v u$. Thus, we need to verify that $\tilde{\mathcal{N}}_{L / M}\left(f_{i}^{\left(L / L_{o}\right)}(v)\right)=\tilde{\mathcal{N}}_{L / M}\left(f_{i}^{\left(L / L_{o}\right)}(v u)\right)$. That is, for each $1 \leq j \in \mathbb{Z}$, we need to check the relation

$$
\begin{gathered}
\prod_{0 \leq \ell \leq f(L / M)-1}\left(\varphi^{d^{\prime}}\right)^{\ell} \widetilde{N}_{E_{j} / E_{j} \cap M}\left(\operatorname{Pr}_{\widetilde{E}_{j}}\left(f_{i}^{\left(L / L_{o}\right)}(v)\right)\right) \\
=\prod_{0 \leq \ell \leq f(L / M)-1}\left(\varphi^{d^{\prime}}\right)^{\ell} \widetilde{N}_{E_{j} / E_{j} \cap M}\left(\operatorname{Pr}_{\widetilde{E}_{j}}\left(f_{i}^{\left(L / L_{o}\right)}(v u)\right)\right) .
\end{gathered}
$$

For $j>i$, we have

$$
\begin{aligned}
\prod_{0 \leq \ell \leq f(L / M)-1}\left(\varphi^{d^{\prime}}\right)^{\ell} \widetilde{N}_{E_{j} / E_{j} \cap M}\left(\operatorname{Pr}_{\widetilde{E}_{j}}\left(f_{i}^{\left(L / L_{o}\right)}(v)\right)\right) \\
=\prod_{0 \leq \ell \leq f(L / M)-1}\left(\varphi^{d^{\prime}}\right)^{\ell} \widetilde{N}_{E_{j} / E_{j} \cap M}\left(g_{j-1}^{\left(L / L_{o}\right)} \circ \cdots \circ g_{i}^{\left(L / L_{o}\right)}(v)\right) \\
=g_{j-1}^{\left(M / M_{o}\right)} \circ \cdots \circ g_{i}^{\left(M / M_{o}\right)}\left(\prod_{0 \leq \ell \leq f(L / M)-1}\left(\varphi^{d^{\prime}}\right)^{\ell} \widetilde{N}_{E_{i} / E_{i} \cap M}(v)\right) \\
=g_{j-1}^{\left(M / M_{o}\right)} \circ \cdots \circ g_{i}^{\left(M / M_{o}\right)}\left(\prod_{0 \leq \ell \leq f(L / M)-1}\left(\varphi^{d^{\prime}}\right)^{\ell} \widetilde{N}_{E_{i} / E_{i} \cap M}(v u)\right) \\
=\prod_{0 \leq \ell \leq f(L / M)-1}\left(\varphi^{d^{\prime}}\right)^{\ell} \widetilde{N}_{E_{j} / E_{j} \cap M}\left(g_{j-1}^{\left(L / L_{o}\right)} \circ \cdots \circ g_{i}^{\left(L / L_{o}\right)}(v u)\right) \\
=\prod_{0 \leq \ell \leq f(L / M)-1}\left(\varphi^{d^{\prime}}\right)^{\ell} \widetilde{N}_{E_{j} / E_{j} \cap M}\left(\operatorname{Pr}_{\widetilde{E}_{j}}\left(f_{i}^{\left(L / L_{o}\right)}(v u)\right)\right) .
\end{aligned}
$$

Thus, the map

$$
f_{i}^{\left(M / M_{o}\right)}: U_{\overparen{E_{i} \cap M}}^{\left.\sigma_{i}\right|_{\widetilde{M}}-1} \rightarrow U_{\widetilde{\mathbb{X}}(M / K)}
$$

is well defined. Moreover, for $j>i$ we have

$$
\operatorname{Pr} \widetilde{E_{j} \cap M} \circ f_{i}^{\left(M / M_{o}\right)}=\left.\left(g_{j-1}^{\left(M / M_{o}\right)} \circ \cdots \circ g_{i}^{\left(M / M_{o}\right)}\right)\right|_{U_{\overparen{E_{i} \cap M}}^{\left.\sigma_{i}\right|_{\widetilde{M}} ^{-1}}} .
$$

Indeed, for $w \in U_{\widetilde{E_{i} \cap M}}^{\left.\sigma_{i}\right|_{\widetilde{M}}-1}$, there exists $v \in U_{\widetilde{E}_{i}}^{\sigma_{i}-1}$ such that

$$
\prod_{0 \leq \ell \leq f(L / M)-1}\left(\varphi^{d^{\prime}}\right)^{\ell} \widetilde{N}_{E_{i} / E_{i} \cap M}(v)=w
$$

and

$$
f_{i}^{\left(M / M_{o}\right)}(w)=\tilde{\mathcal{N}}_{L / M}\left(f_{i}^{\left(L / L_{o}\right)}(v)\right)
$$


That is, the square

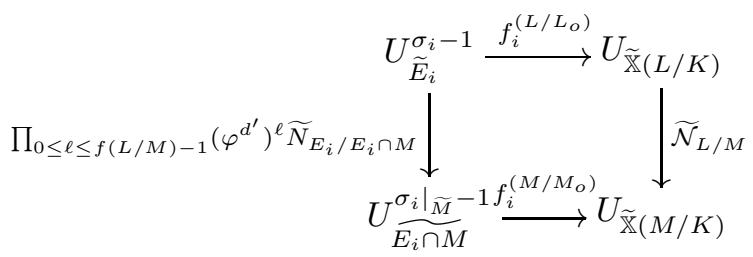

is commutative. Thus,

$$
\begin{aligned}
\operatorname{Pr}_{E_{j} \cap M} \circ f_{i}^{\left(M / M_{o}\right)}(w)=\operatorname{Pr} \widetilde{E_{j} \cap M} \circ \widetilde{\mathcal{N}}_{L / M}\left(f_{i}^{\left(L / L_{o}\right)}(v)\right) \\
\quad=\prod_{0 \leq \ell \leq f(L / M)-1}\left(\varphi^{d^{\prime}}\right)^{\ell} \widetilde{N}_{E_{j} / E_{j} \cap M}\left(\operatorname{Pr}_{\widetilde{E}_{j}} \circ f_{i}^{\left(L / L_{o}\right)}(v)\right) \\
\quad=\prod_{0 \leq \ell \leq f(L / M)-1}\left(\varphi^{d^{\prime}}\right)^{\ell} \widetilde{N}_{E_{j} / E_{j} \cap M}\left(\left(g_{j-1}^{\left(L / L_{o}\right)} \circ \cdots \circ g_{i}^{\left(L / L_{o}\right)}\right)(v)\right) \\
\quad=\left(g_{j-1}^{\left(M / M_{o}\right)} \circ \cdots \circ g_{i}^{\left(M / M_{o}\right)}\right)\left(\prod_{0 \leq \ell \leq f(L / M)-1}\left(\varphi^{d^{\prime}}\right)^{\ell} \widetilde{N}_{E_{i} / E_{i} \cap M}(v)\right),
\end{aligned}
$$

which is the desired relation.

Now, we modify Lemma 5.28 of 8 and show that the norm map $\widetilde{\mathcal{N}}_{L / M}: \widetilde{\mathbb{X}}(L / K)^{\times} \rightarrow$ $\widetilde{\mathbb{X}}(M / K)^{\times}$introduced in (2.17) and (2.18) possesses the following properties.

Lemma 2.21. For the norm map $\widetilde{\mathcal{N}}_{L / M}: \widetilde{\mathbb{X}}(L / K)^{\times} \rightarrow \widetilde{\mathbb{X}}(M / K)^{\times}$introduced by (2.17) and (2.18), we have

(i) $\tilde{\mathcal{N}}_{L / M}\left(Z_{L / L_{0}}\left(\left\{E_{i}, f_{i}^{\left(L / L_{o}\right)}\right\}\right)\right) \subseteq Z_{M / M_{0}}\left(\left\{E_{i} \cap M, f_{i}^{\left(M / M_{o}\right)}\right\}\right)$;

(ii) $\widetilde{\mathcal{N}}_{L / M} \circ\langle\varphi\rangle_{L / M}\left(Y_{L / L_{0}}\right) \subseteq Y_{M / M_{0}}$.

Proof. (i) Recall that $\widetilde{\mathcal{N}}_{L / M}: \widetilde{\mathbb{X}}(L / K)^{\times} \rightarrow \widetilde{\mathbb{X}}(M / K)^{\times}$is a continuous mapping. Now, for any choice of $z^{(i)} \in \operatorname{im}\left(f_{i}^{\left(L / L_{o}\right)}\right)$, the continuity of the multiplicative arrow $\widetilde{\mathcal{N}}_{L / M}$ : $\widetilde{\mathbb{X}}(L / K)^{\times} \rightarrow \widetilde{\mathbb{X}}(M / K)^{\times}$yields

$$
\widetilde{\mathcal{N}}_{L / M}\left(\prod_{i} z^{(i)}\right)=\prod_{i} \widetilde{\mathcal{N}}_{L / M}\left(z^{(i)}\right),
$$

where $\widetilde{\mathcal{N}}_{L / M}\left(z^{(i)}\right) \in \operatorname{im}\left(f_{i}^{\left(M / M_{o}\right)}\right)$ by the commutative square (2.46).

(ii) For $y \in Y_{L / L_{0}}$, since $y^{1-\varphi^{d}} \in Z_{L / L_{0}}$, it follows that $\widetilde{\mathcal{N}}_{L / M}\left(y^{1-\varphi^{d}}\right) \in Z_{M / M_{0}}$ by part (i). Observe that

$$
\widetilde{\mathcal{N}}_{L / M}\left(y^{1-\varphi^{d}}\right)=\widetilde{\mathcal{N}}_{L / M}(y)^{1-\varphi^{d}}=\left(\widetilde{\mathcal{N}}_{L / M}(y)^{1+\varphi^{d^{\prime}}+\cdots+\varphi^{d^{\prime}(f(L / M)-1)}}\right)^{1-\varphi^{d^{\prime}}} .
$$

Therefore,

$$
\widetilde{\mathcal{N}}_{L / M}(y)^{1+\varphi^{d^{\prime}}+\cdots+\varphi^{d^{\prime}(f(L / M)-1)}}=\widetilde{\mathcal{N}}_{L / M} \circ\langle\varphi\rangle_{L / M}(y) \in Y_{M / M_{0}}
$$

as desired.

Part (ii) of Lemma 2.21 shows that the homomorphism $\widetilde{\mathcal{N}}_{L / M} \circ\langle\varphi\rangle_{L / M}: \widetilde{\mathbb{X}}(L / K)^{\times} \rightarrow$ $\widetilde{\mathbb{X}}(M / K)^{\times}$induces a group homomorphism, which will again be called the Coleman norm map from $L$ to $M$; this homomorphism

$$
\tilde{\mathcal{N}}_{L / M}^{\text {Coleman }}: U_{\widetilde{\mathbb{X}}(L / K)}^{\diamond} / Y_{L / L_{0}} \rightarrow U_{\widetilde{\mathbb{X}}(M / K)}^{\diamond} / Y_{M / M_{0}}
$$


is defined by the formula

$$
\widetilde{\mathcal{N}}_{L / M}^{\text {Coleman }}(\bar{U})=\widetilde{\mathcal{N}}_{L / M} \circ\langle\varphi\rangle_{L / M}(U) \cdot Y_{M / M_{0}}
$$

for every $U \in U_{\widetilde{\mathbb{X}}(L / K)}^{\diamond}$, where, as usual, $\bar{U}$ denotes the coset $U . Y_{L / L_{0}}$ in $U_{\widetilde{\mathbb{X}}(L / K)}^{\diamond} / Y_{L / L_{0}}$.

The following lemma is a refinement of Lemma 2.10.

Lemma 2.22. Let $K$ be a local field satisfying condition (2.34). For an infinite Galois subextension $M / K$ of $L / K$ such that the residue-class degree $\left[\kappa_{M}: \kappa_{K}\right]$ is equal to $d^{\prime}$ and $K \subset M \subset K_{\varphi^{d^{\prime}}}$ for some $d^{\prime} \mid d$, the following square is commutative:

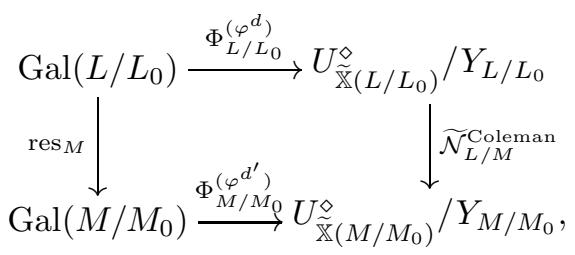

where the right vertical arrow is the Coleman norm map $\widetilde{\mathcal{N}}_{L / M}^{\text {Coleman }}$ from $L$ to $M$ defined by (2.47) and (2.48).

Proof. It suffices to prove that the square

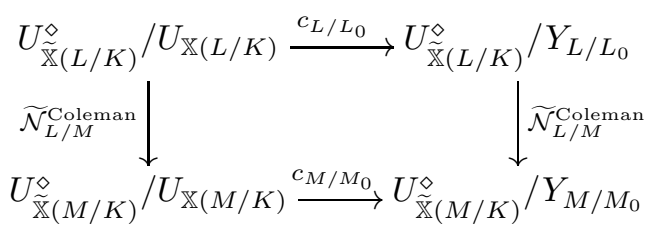

is commutative, which is obvious. Then, uniting this square with the square (2.24), we obtain

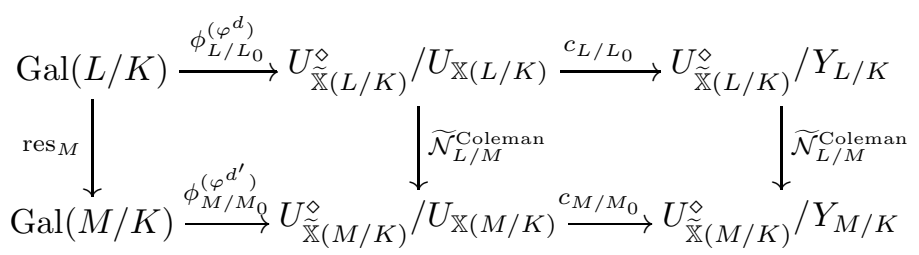

and the commutativity of the square (2.49) follows.

Thus, we have the following theorem, which is a refinement of Theorem 2.11

Theorem 2.23. Let $K$ be a local field satisfying condition (2.34). For an infinite Galois subextension $M / K$ of $L / K$ such that the residue-class degree $\left[\kappa_{M}: \kappa_{K}\right]$ is equal to $d^{\prime}$ and $K \subset M \subset K_{\varphi^{d^{\prime}}}$ for some $d^{\prime} \mid d$, the following square is commutative:

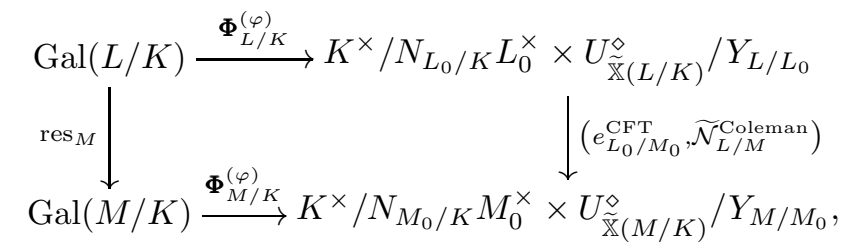

where the right-vertical arrow

$$
K^{\times} / N_{L_{0} / K} L_{0}^{\times} \times U_{\widetilde{\mathbb{X}}(L / K)}^{\diamond} / Y_{L / L_{0}} \stackrel{\left(e_{L_{0} / M_{0}}^{\mathrm{CFT}} \widetilde{\mathcal{N}}_{L / M}^{\text {Coleman }}\right)}{\longrightarrow} K^{\times} / N_{M_{0} / K} M_{0}^{\times} \times U_{\widetilde{\mathbb{X}}(M / K)}^{\diamond} / Y_{M / M_{0}}
$$


is defined by

$$
\left(e_{L_{0} / M_{0}}^{\mathrm{CFT}}, \tilde{\mathcal{N}}_{L / M}^{\text {Coleman }}\right):(\bar{a}, \bar{U}) \mapsto\left(e_{L_{0} / M_{0}}^{\mathrm{CFT}}(\bar{a}), \tilde{\mathcal{N}}_{L / M}^{\text {Coleman }}(\bar{U})\right)
$$

for every $(\bar{a}, \bar{U}) \in K^{\times} / N_{L_{0} / K} L_{0}^{\times} \times U_{\widetilde{\mathbb{X}}(L / K)}^{\diamond} / Y_{L / L_{0}}$. Here

$$
e_{L_{0} / M_{0}}^{\mathrm{CFT}}: K^{\times} / N_{L_{0} / K} L_{0}^{\times} \rightarrow K^{\times} / N_{M_{0} / K} M_{0}^{\times}
$$

is the natural inclusion defined via the existence theorem of the local class field theory.

Proof. By the isomorphism defined by (2.1) and (2.2), for $\sigma \in \operatorname{Gal}(L / K)$ there exists a unique $0 \leq m \in \mathbb{Z}$ such that $\left.\sigma\right|_{L_{0}}=\varphi^{m}$ and $\varphi^{-m} \sigma \in \operatorname{Gal}\left(L / L_{0}\right)$. Now, by definition,

$$
\Phi_{L / K}^{(\varphi)}(\sigma)=\left(\pi_{K}^{m} N_{L_{0} / K} L_{0}^{\times}, \Phi_{L / L_{0}}^{\left(\varphi^{d}\right)}\left(\varphi^{-m} \sigma\right)\right) .
$$

Thus,

$$
\begin{aligned}
& \left(e_{L_{0} / M_{0}}^{\mathrm{CFT}}, \widetilde{\mathcal{N}}_{L / M}^{\text {Coleman }}\right)\left(\pi_{K}^{m} N_{L_{0} / K} L_{0}^{\times}, \Phi_{L / L_{0}}^{\left(\varphi^{d}\right)}\left(\varphi^{-m} \sigma\right)\right) \\
& \quad=\left(e_{L_{0} / M_{0}}^{\mathrm{CFT}}\left(\pi_{K}^{m} N_{L_{0} / K} L_{0}^{\times}\right), \widetilde{\mathcal{N}}_{L / M}^{\text {Coleman }}\left(\Phi_{L / L_{0}}^{\left(\varphi^{d}\right)}\left(\varphi^{-m} \sigma\right)\right)\right) \\
& \quad=\left(\pi_{K}^{m} N_{M_{0} / K} M_{0}^{\times}, \Phi_{M / M_{0}}^{\left(\varphi^{d^{\prime}}\right)}\left(\left.\varphi^{-m} \sigma\right|_{M}\right)\right)
\end{aligned}
$$

by Lemma 2.22. Note that, by the existence theorem of local class field theory, we have

$$
e_{L_{0} / M_{0}}^{\mathrm{CFT}}\left(\pi_{K}^{m} N_{L_{0} / K} L_{0}^{\times}\right)=\pi_{K}^{m} N_{M_{0} / K} M_{0}^{\times}=\pi_{K}^{m^{\prime}} N_{M_{0} / K} M_{0}^{\times},
$$

where $0 \leq m^{\prime} \in \mathbb{Z}$ is a unique integer satisfying $\left.\left(\left.\sigma\right|_{M}\right)\right|_{M_{0}}=\left.\sigma\right|_{M_{0}}=\varphi^{m^{\prime}}$ and $\varphi^{-m^{\prime}}\left(\left.\sigma\right|_{M}\right)$ $\in \operatorname{Gal}\left(M / M_{0}\right)$. Hence,

$$
\begin{aligned}
\left(e_{L_{0} / M_{0}}^{\mathrm{CFT}}, \widetilde{\mathcal{N}}_{L / M}^{\text {Coleman }}\right) & \left(\Phi_{L / K}^{(\varphi)}(\sigma)\right)=\left(\pi_{K}^{m^{\prime}} N_{M_{0} / K} M_{0}^{\times}, \Phi_{M / M_{0}}^{\left(\varphi^{d^{\prime}}\right)}\left(\left.\varphi^{-m} \sigma\right|_{M}\right)\right) \\
& =\left(\pi_{K}^{m^{\prime}} N_{M_{0} / K} M_{0}^{\times}, \Phi_{M / M_{0}}^{\left(\varphi^{d^{\prime}}\right)}\left(\varphi^{-m^{\prime}}(\sigma \mid M)\right)\right)=\boldsymbol{\Phi}_{M / K}^{(\varphi)}\left(\operatorname{res}_{M}(\sigma)\right)
\end{aligned}
$$

by Remark 2.2, part (i), which completes the proof.

Let $K$ be a local field satisfying condition (2.34). Let $F / K$ be a finite subextension of $L / K$. Thus, $L / F$ is an infinite APF Galois extension (see [8, Lemma 3.3]), where $F$ satisfies (2.34). Fix a Lubin-Tate splitting $\varphi_{F}$ over $F$ and assume that the residue-class degree $\left[\kappa_{L}: \kappa_{F}\right]$ is equal to $d^{\prime}$ for some $d^{\prime} \mid d$ and that there exists a chain of field extensions

$$
F \subset L \subset F_{\left(\varphi_{F}\right)^{d^{\prime}}}
$$

Then we have the generalized Fesenko reciprocity map

$$
\boldsymbol{\Phi}_{L / F}^{\left(\varphi_{F}\right)}: \operatorname{Gal}(L / F) \rightarrow F^{\times} / N_{L_{0}^{(F)} / F} L_{0}^{(F)^{\times}} \times U_{\widetilde{\mathbb{X}}(L / F)}^{\diamond} / Y_{L / L_{0}^{(F)}}
$$

corresponding to the extension $L / F$. Here, as usual, $L_{0}^{(F)}$ is defined by $L_{0}^{(F)}=L \cap F^{n r}=$ $F_{d^{\prime}}^{n r}$ (we recall that $L_{0}^{(K)}=L \cap K^{n r}=K_{d}^{n r}$ ).

Now, we fix a basic sequence

$$
L_{0}^{(K)}=E_{o} \subset E_{1} \subset \cdots \subset E_{i} \subset \cdots \subset L
$$

for the extension $L / L_{0}^{(K)}$. Using the notation of [3] and [8], for each $1 \leq i \in \mathbb{Z}$ we introduce an element $\sigma_{i}$ in $\operatorname{Gal}(\widetilde{L} / \widetilde{K})$ such that $\left\langle\left.\sigma\right|_{E_{i}}\right\rangle=\operatorname{Gal}\left(E_{i} / E_{i-1}\right)$. Next, we fix the sequence

$$
L_{0}^{(F)}=E_{o} L_{0}^{(F)} \subseteq E_{1} L_{0}^{(F)} \subseteq \cdots \subseteq E_{i} L_{0}^{(F)} \subseteq \cdots \subseteq L L_{0}^{(F)}=L
$$


for the extension, $L / L_{0}^{(F)}$, as in [8, (5.55)]. For $1 \leq i \in \mathbb{Z}$, we introduce elements $\sigma_{i}^{*}$ in $\operatorname{Gal}(\widetilde{L} / \widetilde{F})$ that satisfy

$$
\left\langle\left.\sigma_{i}^{*}\right|_{E_{i} L_{0}^{(F)}}\right\rangle=\operatorname{Gal}\left(E_{i} L_{0}^{(F)} / E_{i-1} L_{0}^{(F)}\right)
$$

as follows:

(i) for $i>i_{o}$ we put $\sigma_{i}^{*}=\sigma_{i}$;

(ii) for $i \leq i_{o}$ we put

$$
\sigma_{i}^{*}= \begin{cases}\sigma_{i} & \text { if } E_{i-1} L_{0}^{(F)} \subset E_{i} L_{0}^{(F)}, \\ \operatorname{id}_{E_{i} L_{0}^{(F)}} & \text { if } E_{i-1} L_{0}^{(F)}=E_{i} L_{0}^{(F)}\end{cases}
$$

where $i_{o}$ is defined as in $[8$, (5.55)]. Then it is clear that, for each $1 \leq i \in \mathbb{Z}$, the elements $\sigma_{i}^{*}$ of $\operatorname{Gal}(\widetilde{L} / \widetilde{F})$ satisfy

$$
\left\langle\left.\sigma_{i}^{*}\right|_{E_{i} L_{0}^{(F)}}\right\rangle=\operatorname{Gal}\left(E_{i} L_{0}^{(F)} / E_{i-1} L_{o}^{(F)}\right),
$$

and that $\sigma_{i}^{*}=\sigma_{i}$ for almost all $i$. Next, for all $1 \leq k, i \in \mathbb{Z}$, we introduce the map

$$
h_{k}^{\left(L / L_{0}^{(F)}\right)}: \prod_{1 \leq i \leq k} \frac{U_{i}^{\sigma_{i}^{*}-1}}{E_{k} L_{0}^{(F)}} \rightarrow\left(\prod_{1 \leq i \leq k+1} \frac{U^{\sigma_{i}^{*}-1}}{E_{k+1} L_{0}^{(F)}}\right) / U_{E_{k+1}^{\sigma_{k+1} L_{0}^{(F)}}}^{\sigma^{*}-1},
$$

the map

$$
g_{k}^{\left(L / L_{0}^{(F)}\right)}: \prod_{1 \leq i \leq k} U^{\sigma_{i}^{*}-1} \rightarrow \prod_{E_{k} L_{0}^{(F)}} U_{1 \leq i \leq k+1}^{U^{\sigma_{i}^{*}-1}}
$$

and the map

$$
f_{i}^{\left(L / L_{0}^{(F)}\right)}: U_{E_{i} L_{0}^{(F)}}^{\sigma_{i}^{*}-1} \rightarrow U_{\widetilde{\mathbb{X}}\left(L / E_{i} L_{0}^{(F)}\right)} \stackrel{\Lambda_{E_{i} L_{0}^{(F)} / L_{0}^{(F)}}}{\longrightarrow} U_{\widetilde{\mathbb{X}}(L / F)}
$$

as in [3] and [8]. For each $1 \leq k \in \mathbb{Z}$, we define a homomorphism

$$
h_{k}^{\left(L / L_{0}^{(K)}\right)}: \prod_{1 \leq i \leq k} U_{\widetilde{E}_{k}}^{\sigma_{i}-1} \rightarrow\left(\prod_{1 \leq i \leq k+1} U_{\widetilde{E}_{k+1}^{\sigma_{i}-1}}\right) / U_{\widetilde{E}_{k+1}}^{\sigma_{k+1}-1}
$$

that satisfies

$$
\widetilde{N}_{E_{k+1} L_{0}^{(F)} / E_{k+1}}^{*} \circ h_{k}^{\left(L / L_{0}^{(F)}\right)}=h_{k}^{\left(L / L_{0}^{(K)}\right)} \circ \widetilde{N}_{E_{k} L_{0}^{(F)} / E_{k}}
$$

and take any map

$$
g_{k}^{\left(L / L_{0}^{(K)}\right)}: \prod_{1 \leq i \leq k} U_{\widetilde{E}_{k}}^{\sigma_{i}-1} \rightarrow \prod_{1 \leq i \leq k+1} U_{\widetilde{E}_{k+1}^{\sigma_{i}-1}}^{\sigma^{(K)}}
$$

that satisfies

$$
\widetilde{N}_{E_{k+1} L_{0}^{(F)} / E_{k+1}} \circ g_{k}^{\left(L / L_{0}^{(F)}\right)}=g_{k}^{\left(L / L_{0}^{(K)}\right)} \circ \widetilde{N}_{E_{k} L_{0}^{(F)} / E_{k}},
$$

again following the same lines of [3] and [8].

Now, for each $1 \leq i \in \mathbb{Z}$, we introduce the map $f_{i}^{\left(L / L_{0}^{(K)}\right)}: U_{\widetilde{E}_{i}}^{\sigma_{i}-1} \rightarrow U_{\widetilde{\mathbb{X}}(L / K)}$ by $f_{i}^{\left(L / L_{0}^{(K)}\right)}(w)=\Lambda_{F / K}\left(f_{i}^{\left(L / L_{0}^{(F)}\right)}(v)\right)$, where $v \in U_{E_{i}^{L_{0}^{(F)}}}^{\sigma^{*}-1}$ is any element satisfying $\widetilde{N}_{E_{i} L_{0}^{(F)} / E_{i}}(v)=w \in U_{\widetilde{E}_{i}}^{\sigma_{i}-1}$. Note that if $v^{\prime} \in U_{E_{i} L_{0}^{*}-1}^{E_{0}^{(F)}}$ is such that $\widetilde{N}_{E_{i} L_{0}^{(F)} / E_{i}}\left(v^{\prime}\right)=w$, then $\Lambda_{F / K}\left(f_{i}^{\left(L / L_{0}^{(F)}\right)}(v)\right)=\Lambda_{F / K}\left(f_{i}^{\left(L / L_{0}^{(F)}\right)}\left(v^{\prime}\right)\right)$. 
Indeed, there exists $u \in \operatorname{ker}\left(\widetilde{N}_{E_{i} L_{0}^{(F)} / E_{i}}\right)$ such that $v^{\prime}=v u$. Thus, we need to verify that $\Lambda_{F / K}\left(f_{i}^{\left(L / L_{0}^{(F)}\right)}(v)\right)=\Lambda_{F / K}\left(f_{i}^{\left(L / L_{0}^{(F)}\right)}(v u)\right)$. That is, for each $1 \leq j \in \mathbb{Z}$, we need to check the relation

$$
\operatorname{Pr}_{\widetilde{E}_{j}}\left(\Lambda_{F / K}\left(f_{i}^{\left(L / L_{0}^{(F)}\right)}(v)\right)\right)=\operatorname{Pr}_{\widetilde{E}_{j}}\left(\Lambda_{F / K}\left(f_{i}^{\left(L / L_{0}^{(F)}\right)}(v u)\right)\right) .
$$

For $j>i$, we have

$$
\begin{aligned}
\operatorname{Pr}_{\widetilde{E}_{j}}\left(\Lambda_{F / K}\left(f_{i}^{\left(L / L_{0}^{(F)}\right)}(v)\right)\right) & =\widetilde{N}_{E_{j} L_{0}^{(F)} / E_{j}}\left(\operatorname{Pr} \widetilde{E_{j} L_{0}^{(F)}}\left(\Lambda_{F / K}\left(f_{i}^{\left(L / L_{0}^{(F)}\right)}(v)\right)\right)\right) \\
& =\widetilde{N}_{E_{j} L_{0}^{(F)} / E_{j}}\left(\operatorname{Pr}_{E_{j} L_{0}^{(F)}}\left(f_{i}^{\left(L / L_{0}^{(F)}\right)}(v)\right)\right) \\
& =\widetilde{N}_{E_{j} L_{0}^{(F)} / E_{j}}\left(g_{j-1}^{\left(L / L_{0}^{(F)}\right)} \circ \cdots \circ g_{i}^{\left(L / L_{0}^{(F)}\right)}(v)\right) \\
& =g_{j-1}^{\left(L / L_{0}^{(K)}\right)} \circ \cdots \circ g_{i}^{\left(L / L_{0}^{(K)}\right)}\left(\widetilde{N}_{E_{i} L_{0}^{(F)} / E_{i}}(v)\right),
\end{aligned}
$$

by the properties of the mappings $g_{k}^{\left(L / L_{0}^{(F)}\right)}$ and $g_{k}^{\left(L / L_{0}^{(K)}\right)}$. Thus, relation (2.50) follows, because $\widetilde{N}_{E_{i} L_{0}^{(F)} / E_{i}}(v)=\widetilde{N}_{E_{i} L_{0}^{(F)} / E_{i}}(v u)$. Therefore, the map

$$
f_{i}^{\left(L / L_{0}^{(K)}\right)}: U_{\widetilde{E}_{i}}^{\sigma_{i}-1} \rightarrow U_{\widetilde{\mathbb{X}}(L / K)}
$$

is well defined. Moreover, for $j>i$, we have

$$
\operatorname{Pr}_{\widetilde{E}_{j}} \circ f_{i}^{\left(L / L_{0}^{(K)}\right)}=\left.\left(g_{j-1}^{\left(L / L_{0}^{(K)}\right)} \circ \cdots \circ g_{i}^{\left(L / L_{0}^{(K)}\right)}\right)\right|_{U_{\widetilde{E}_{i}}^{\sigma_{i}-1}} .
$$

Indeed, for $w \in U_{\widetilde{E}_{i}}^{\sigma_{i}-1}$, there exists $v \in U_{E_{i} L_{0}^{(F)}}^{\sigma_{i}^{*}-1}$ such that $\widetilde{N}_{E_{i} L_{0}^{(F)} / E_{i}}(v)=w$, and $f_{i}^{\left(L / L_{0}^{(K)}\right)}(w)=\Lambda_{F / K}\left(f_{i}^{\left(L / L_{0}^{(F)}\right)}(v)\right)$. That is, the square

$$
\begin{aligned}
& U_{E_{i} L_{0}^{(F)}}^{\sigma_{i}^{*}-1} f_{i}^{\left(L / L_{0}^{(F)}\right)} U_{\widetilde{\mathbb{X}}(L / F)} \\
& \left.\widetilde{N}_{E_{i} L_{0}^{(F)} / E_{i}}\right|_{\substack{U_{\widetilde{E}_{i}}^{\sigma_{i}-1}}} \stackrel{f_{i}^{\left(L / L_{0}^{(K)}\right)}}{\longrightarrow} U_{\widetilde{\mathbb{X}}(L / K)} \Lambda_{F / K}
\end{aligned}
$$

is commutative. Thus,

$$
\begin{aligned}
\operatorname{Pr}_{\widetilde{E}_{j}} \circ f_{i}^{\left(L / L_{0}^{(K)}\right)}(w) & =\operatorname{Pr}_{\widetilde{E}_{j}} \circ \Lambda_{F / K}\left(f_{i}^{\left(L / L_{0}^{(F)}\right)}(v)\right) \\
& =\widetilde{N}_{E_{j} L_{0}^{(F)} / E_{j}}\left(\operatorname{Pr} \widetilde{E_{j} L_{0}^{(F)}} \circ f_{i}^{\left(L / L_{0}^{(F)}\right)}(v)\right) \\
& =\widetilde{N}_{E_{j} L_{0}^{(F)} / E_{j}}\left(\left(g_{j-1}^{\left(L / L_{0}^{(F)}\right)} \circ \cdots \circ g_{i}^{\left(L / L_{0}^{(F)}\right)}\right)(v)\right) \\
& =\left(g_{j-1}^{\left(L / L_{0}^{(K)}\right)} \circ \cdots \circ g_{i}^{\left(L / L_{0}^{(K)}\right)}\right)\left(\widetilde{N}_{E_{i} L_{0}^{(F)} / E_{i}}(v)\right),
\end{aligned}
$$

by the properties of the mappings $g_{k}^{\left(L / L_{0}^{(F)}\right)}$ and $g_{k}^{\left(L / L_{0}^{(K)}\right)}$, as claimed.

Now, we modify Lemma 5.30 of $[8]$ and show that the homomorphism

$$
\Lambda_{F / K}: \widetilde{\mathbb{X}}\left(L / L_{0}^{(F)}\right)^{\times} \rightarrow \widetilde{\mathbb{X}}\left(L / L_{0}^{(K)}\right)^{\times}
$$

introduced in (2.25) and (2.26) possesses the following properties. 
Lemma 2.24. For the continuous homomorphism $\Lambda_{F / K}: \widetilde{\mathbb{X}}\left(L / L_{0}^{(F)}\right)^{\times} \rightarrow \widetilde{\mathbb{X}}\left(L / L_{0}^{(K)}\right)^{\times}$ introduced by (2.25) and (2.26) we have

(i) $\Lambda_{F / K}\left(Z_{L / L_{0}^{(F)}}\left(\left\{K_{i} F, f_{i}^{\left(L / L_{0}^{(F)}\right)}\right\}\right)\right) \subseteq Z_{L / L_{0}^{(K)}}\left(\left\{K_{i}, f_{i}^{\left(L / L_{0}^{(K)}\right)}\right\}\right)$;

(ii) $\Lambda_{F / K}\left(Y_{L / L_{0}^{(F)}}\right) \subseteq Y_{L / L_{0}^{(K)}}$.

Proof. (i) For any choice of $z^{(i)} \in Z_{i}^{\left(L / L_{0}^{(F)}\right)}$, the continuity of the multiplicative arrow $\Lambda_{F / K}: \widetilde{\mathbb{X}}\left(L / L_{0}^{(F)}\right)^{\times} \rightarrow \widetilde{\mathbb{X}}\left(L / L_{0}^{(K)}\right)^{\times}$yields

$$
\Lambda_{F / K}\left(\prod_{i} z^{(i)}\right)=\prod_{i} \Lambda_{F / K}\left(z^{(i)}\right)
$$

where $\Lambda_{F / K}\left(z^{(i)}\right) \in Z_{i}^{\left(L / L_{0}^{(K)}\right)}$ by the commutative square (2.51).

(ii) Let $y \in Y_{L / L_{0}^{(F)}}$. Then $y^{1-\varphi_{F}^{d^{\prime}}} \in Z_{L / L_{0}^{(F)}}\left(\left\{K_{i} F, f_{i}^{\left(L / L_{0}^{(F)}\right)}\right\}\right)$. Thus,

$$
\Lambda_{F / K}\left(y^{1-\varphi_{F}^{d^{\prime}}}\right)=\Lambda_{F / K}(y)^{1-\varphi_{F}^{d^{\prime}}} \in Z_{L / L_{0}^{(K)}}\left(\left\{K_{i}, f_{i}^{\left(L / L_{0}^{(K)}\right)}\right\}\right)
$$

by part (i). Now the result follows, because $\varphi_{F}^{d^{\prime}}=\varphi_{K}^{d}$ by Remark 2.12 ,

Thus, the homomorphism $\Lambda_{F / K}: \widetilde{\mathbb{X}}\left(L / L_{0}^{(F)}\right)^{\times} \rightarrow \widetilde{\mathbb{X}}\left(L / L_{0}^{(K)}\right)^{\times}$defined by (2.26) induces a group homomorphism

$$
\lambda_{F / K}: U_{\widetilde{\mathbb{X}}\left(L / L_{0}^{(F)}\right)}^{\diamond} / Y_{L / L_{0}^{(F)}} \rightarrow U_{\widetilde{\mathbb{X}}\left(L / L_{0}^{(K)}\right)}^{\diamond} / Y_{L / L_{0}^{(K)}}
$$

defined by

$$
\lambda_{F / K}(\bar{U})=\Lambda_{F / K}(U) . Y_{L / L_{0}^{(K)}}
$$

for every $U \in U_{\widetilde{\mathbb{X}}\left(L / L_{0}^{(F)}\right)}^{\diamond}$, where, as usual, $\bar{U}$ is the coset $U \cdot Y_{L / L_{0}^{(F)}}$ in $U_{\widetilde{\mathbb{X}}\left(L / L_{0}^{(F)}\right)}^{\diamond} / Y_{L / L_{0}^{(F)}}$.

Let

$$
\boldsymbol{\Phi}_{L / F}^{\left(\varphi_{F}\right)}: \operatorname{Gal}(L / F) \rightarrow F^{\times} / N_{L_{0}^{(F)} / F} L_{0}^{(F)^{\times}} \times U_{\widetilde{\mathbb{X}}\left(L / L_{0}^{(F)}\right)}^{\diamond} / Y_{L / L_{0}^{(F)}}
$$

be the corresponding generalized Fesenko reciprocity map defined for the extension $L / F$, where $Y_{L / L_{0}^{(F)}}=Y_{L / L_{0}^{(F)}}\left(\left\{K_{i} F, f_{i}^{\left(L / L_{0}^{(F)}\right)}\right\}\right)$.

The following lemma is a refinement of Lemma 2.13 .

Lemma 2.25. Let $K$ be a local field satisfying condition (2.34). Let $F / K$ be a finite subextension of $L / K$. Fix a Lubin-Tate splitting $\varphi_{F}$ over $F$ and assume that the residueclass degree $\left[\kappa_{L}: \kappa_{F}\right]$ is equal to $d^{\prime}$ and $F \subset L \subset F_{\left(\varphi_{F}\right)^{\prime}}$ for some $d^{\prime} \mid d$. Then the square

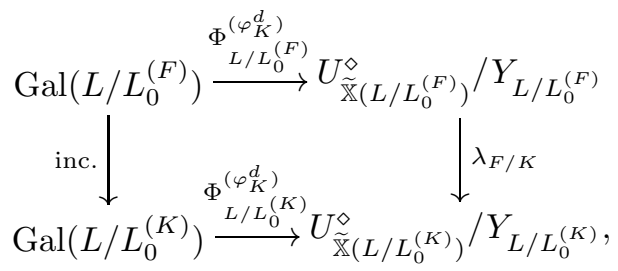

where the right vertical arrow $\lambda_{F / K}: U_{\widetilde{\mathbb{X}}\left(L / L_{0}^{(F)}\right)}^{\diamond} / Y_{L / L_{0}^{(F)}} \rightarrow U_{\widetilde{\mathbb{X}}\left(L / L_{0}^{(K)}\right)} / Y_{L / L_{0}^{(K)}}$ is defined by (2.52) and (2.53), is commutative. 
Proof. It suffices to prove that the square

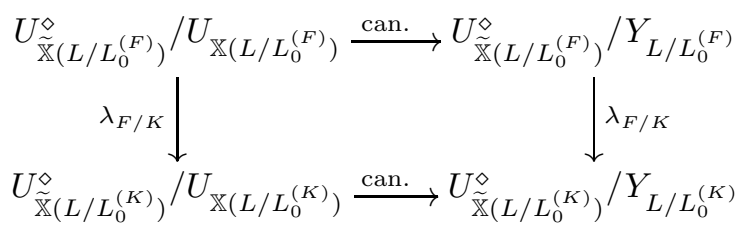

is commutative, which is obvious. Then, uniting this square with the square (2.29), we obtain

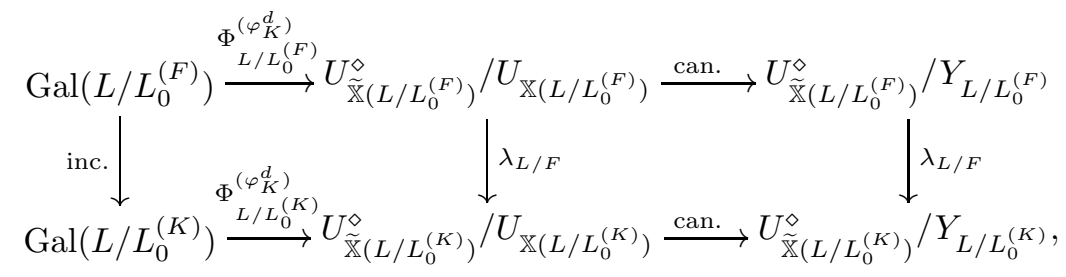

and the commutativity of the square (2.54) follows.

Thus, we have the following theorem, which is a refinement of Theorem 2.14

Theorem 2.26. Let $K$ be a local field satisfying condition (2.34). Let $F / K$ be a finite subextension of $L / K$. Fix a Lubin-Tate splitting $\varphi_{F}$ over $F$ and assume that the residueclass degree $\left[\kappa_{L}: \kappa_{F}\right]$ is equal to $d^{\prime}$ and $F \subset L \subset F_{\left(\varphi_{F}\right)^{\prime}}$ for some $d^{\prime} \mid d$. Then the following square is commutative:

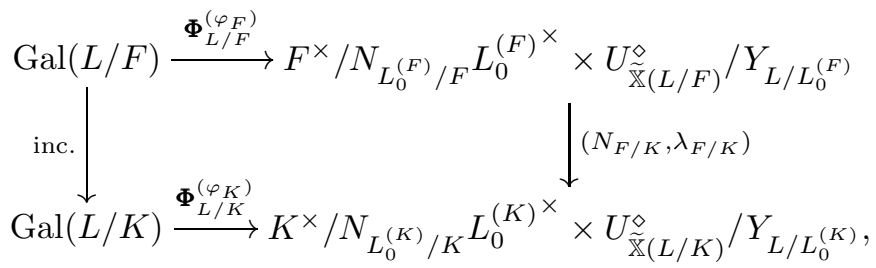

where the right vertical arrow

$$
\begin{aligned}
\left(N_{F / K}, \lambda_{F / K}\right): F^{\times} / N_{L_{0}^{(F)} / F} L_{0}^{(F)^{\times}} \times U_{\widetilde{\mathbb{X}}(L / F)}^{\diamond} / Y_{L / L_{0}^{(F)}} & \\
& \rightarrow K^{\times} / N_{L_{0}^{(K)} / K} L_{0}^{(K)^{\times}} \times U_{\widetilde{\mathbb{X}}(L / K)}^{\diamond} / Y_{L / L_{0}^{(K)}}
\end{aligned}
$$

is defined by $\left(N_{F / K}, \lambda_{F / K}\right):(\bar{a}, \bar{U}) \mapsto\left(\overline{N_{F / K}(a)}, \lambda_{F / K}(\bar{U})\right)$ for every $(\bar{a}, \bar{U})$ belonging to $F^{\times} / N_{L_{0}^{(F)} / F} L_{0}^{(F)^{\times}} \times U_{\widetilde{\mathbb{X}}(L / F)}^{\diamond} / Y_{L / L_{0}^{(F)}}$.

Proof. Let $\sigma \in \operatorname{Gal}(L / F)$. There exists $0 \leq m \in \mathbb{Z}$ such that $\left.\sigma\right|_{L_{0}^{(F)}}=\varphi_{F}^{m}$ and $\varphi_{F}^{-m} \sigma \in$ $\operatorname{Gal}\left(L / L_{0}^{(F)}\right)$. We have

$$
\boldsymbol{\Phi}_{L / F}^{\left(\varphi_{F}\right)}(\sigma)=\left(\pi_{F}^{m} \cdot N_{L_{0}^{(F)} / F} L_{0}^{(F)^{\times}}, \Phi_{L / L_{0}^{(F)}}^{\left(\varphi_{K}^{d}\right)}\left(\varphi_{F}^{-m} \sigma\right)\right)
$$

and

$$
\left(N_{F / K}, \lambda_{F / K}\right)\left(\Phi_{L / F}^{\left(\varphi_{F}\right)}(\sigma)\right)=\left(\pi_{K}^{m} \cdot N_{L_{0}^{(K)} / K} L_{0}^{(K)^{\times}}, \Phi_{L / L_{0}^{(K)}}^{\left(\varphi_{K}^{d}\right)}\left(\varphi_{F}^{-m} \sigma\right)\right)
$$

by the norm-compatibility of primes in the fixed Lubin-Tate labeling and by Lemma 2.25. Now, there exists $0 \leq m^{\prime} \in \mathbb{Z}$ such that $\left.\sigma\right|_{L_{0}^{(K)}}=\varphi_{K}^{m^{\prime}}$ and $\varphi_{K}^{-m^{\prime}} \sigma \in \operatorname{Gal}\left(L / L_{0}^{(K)}\right)$. By part (ii) of Remark 2.2. it follows that $\left.\varphi_{F}^{m}\right|_{L_{0}^{(K)}}=\varphi_{K}^{m^{\prime}}$ and $\varphi_{F}^{-m} \sigma=\varphi_{K}^{-m^{\prime}} \sigma$. By 
the Abelian local class field theory, $N_{F / K}: \pi_{F}^{m} N_{L_{0}^{(F)} / F} L_{0}^{(F)^{\times}} \mapsto \pi_{K}^{m^{\prime}} N_{L_{0}^{(K)} / K} L_{0}^{(K)^{\times}}=$ $\pi_{K}^{m} \cdot N_{L_{0}^{(K)} / K} L_{0}^{(K)^{\times}}$. Thus,

$$
\begin{aligned}
\left(N_{F / K}, \lambda_{F / K}\right)\left(\Phi_{L / F}^{\left(\varphi_{F}\right)}(\sigma)\right) & =\left(\pi_{K}^{m} \cdot N_{L_{0}^{(K)} / K} L_{0}^{(K)^{\times}}, \Phi_{L / L_{0}^{(K)}}^{\left(\varphi_{K}^{d}\right)}\left(\varphi_{F}^{-m} \sigma\right)\right) \\
& =\left(\pi_{K}^{m^{\prime}} \cdot N_{L_{0}^{(K)} / K} L_{0}^{(K)^{\times}}, \Phi_{L / L_{0}^{(K)}}^{\left(\varphi_{K}^{d}\right)}\left(\varphi_{K}^{-m^{\prime}} \sigma\right)\right)=\boldsymbol{\Phi}_{L / K}^{\left(\varphi_{K}\right)}(\sigma),
\end{aligned}
$$

which completes the proof.

Finally, the inverse $\boldsymbol{H}_{L / K}^{(\varphi)}=\left(\boldsymbol{\Phi}_{L / K}^{(\varphi)}\right)^{-1}$ of the generalized Fesenko reciprocity map $\Phi_{L / K}^{(\varphi)}$ defined for the extension $L / K$ is a generalization of the Hazewinkel map for infinite APF Galois subextensions $L / K$ of $K_{\varphi^{d}} / K$ satisfying $\left[\kappa_{L}: \kappa_{K}\right]=d$ and under the assumption that the local field $K$ satisfies condition (2.34). More precisely, the following is true.

Proposition 2.27. The square

$$
\begin{aligned}
& K^{\times} / N_{L_{0} / K} L_{0}^{\times} \times U_{\widetilde{\mathbb{X}}(L / K)}^{\ominus} / Y_{L / L_{0}} \stackrel{\boldsymbol{H}_{L / K}^{(\varphi)}}{\longrightarrow} \operatorname{Gal}(L / K) \\
&\left(\operatorname{id}_{K} \times / N_{L_{0} / K} L_{0}^{\times}, \operatorname{Pr}_{\widetilde{K}}\right) \downarrow \\
& K^{\times} / N_{L_{0} / K} L_{0}^{\times} \times U_{L_{0}} / N_{L / L_{0}} U_{L} \stackrel{h_{L / K}}{\longrightarrow} \operatorname{Gal}(L / K)^{a b} \operatorname{Gal}(L / K)^{\prime}
\end{aligned}
$$

is commutative, where $h_{L / K}: K^{\times} / N_{L_{0} / K} L_{0}^{\times} \times U_{L_{0}} / N_{L / L_{0}} U_{L} \rightarrow \operatorname{Gal}(L / K)^{a b}$ is the Hazewinkel map of $L / K$.

\section{ACKNOWLEDGEMENTS}

The first author is grateful to Institut de Mathématiques, "Théorie des Groupes, Représentations et Applications", Université Paris 7, Jussieu, Paris, France, and to the School of Pure Mathematics of the Tata Institute of Fundamental Research, Mumbai, India, where some parts of this work were completed, for their hospitality and support.

The second named author is grateful to Tübitak for a fellowship, and to the School of Mathematics of the University of Nottingham, where some parts of this work were initiated, for hospitality and support.

Both authors would like to thank I. B. Fesenko for his interest and encouragement at all stages of this work.

\section{REFERENCES}

[1] I. B. Fesenko, Local reciprocity cycles, Invitation to Higher Local Fields (Münster, 1999) (I. B. Fesenko, M. Kurihara, eds.), Geom. Topol. Monogr., vol. 3, Geom. Topol. Publ., Coventry, 2000, pp. 293-298. MR.1804942(2001k:11239)

[2] _ Nonabelian local reciprocity maps, Class Field Theory - Its Centenary and Prospect (Tokyo, 1998) (K. Miyake, ed.), Adv. Stud. Pure Math., vol. 30, Math. Soc. Japan, Tokyo, 2001, pp. 63-78. MR:1846451 (2002f:11177)

[3] _ On the image of noncommutative local reciprocity map, Homology, Homotopy Appl. 7 (2005), 53-62. MR2200206 (2006m:11171)

[4] I. B. Fesenko and S. V. Vostokov, Local fields and their extensions. A constructive approach, Transl. Math. Monogr., vol. 121, Amer. Math. Soc., Providence, RI, 1993. MR1218392 (94d:11095)

[5] J.-M. Fontaine and J.-P. Wintenberger, Le "corps des normes" de certaines extensions algébriques de corps locaux, C. R. Acad. Sci. Paris Sér. A Math. 288 (1979), 367-370. MR0526137 (80b:12015)

[6] Extensions algébriques et corps des normes des extensions APF des corps locaux, C. R. Acad. Sci. Paris Sér. A Math. 288 (1979), 441-444. MR0527692 (80h:12014)

[7] A. Gurevich, Ph.D. Thesis, Humboldt Univ., Berlin, 1997. 
[8] K. I. Ikeda and E. Serbest, Fesenko reciprocity map, Algebra i Analiz 20 (2008), no. 3, 112-162; English transl. in St. Petersburg Math. J. 20 (2009), no. 3. MR.2454454

[9] Non-abelian local class field theory, Preprint, 2007.

[10] H. Koch and E. de Shalit, Metabelian local class field theory, J. Reine Angew. Math. 478 (1996), 85-106. MR:1409054 (97f:11095)

[11] F. Laubie, Une théorie du corps de classes local non abélien, Compositio Math. 143 (2007), 339362. MR2309990 (2008b:11124)

[12] J.-P. Wintenberger, Le corps des normes de certaines extensions infinies de corps locaux; applications, Ann. Sci. École Norm. Sup. (4) 46 (1983), 59-89. MR0719763 (85e:11098)

Department of Mathematics, Yeditepe University, 26 AĞustos Yerleşimi, İnönü Mah., KAYIŞDaĞI CAD., 34755 KadikÖy, Istanbul, TuRkey

E-mail address: ilhan.ikeda@yeditepe.edu.tr

Gümüş Pala Mahallesi, Gümüş Sok., Öz Aksu Sitesi, C-2/39, 34160 Avcilar, Istanbul, Turkey E-mail address: erols730yahoo.com

Received 20/OCT/2007

Originally published in English 